\title{
On the Long Time Behavior of the Doubly Infinite Toda Lattice under Initial Data Decaying at Infinity
}

\author{
Spyridon Kamvissis
}

Ecole Normale Supérieure, Lyons, France

Received May 18, 1992; in revised form August 18, 1992

\begin{abstract}
We provide rigorous analysis of the long time behavior of the (doubly infinite) Toda lattice under initial data that decay at infinity, in the absence of solitons. We solve (approximately and for large times) the Riemann-Hilbert matrix factorization problem equivalent to the related inverse scattering problem with the help of the Beals-Coifman formula, by reducing it to a simpler one through a series of contour deformations in the spirit of the Deift-Zhou method.
\end{abstract}

\section{Introduction}

\subsection{Statement of the Problem and Results}

In this paper we consider the doubly infinite Toda lattice under initial conditions decaying at infinite. More precisely, we provide the full analysis of the long-time behavior of the initial value problem:

$$
\begin{aligned}
& \dot{a}_{n}=2\left(b_{n}^{2}-b_{n-1}^{2}\right), \quad \infty<n<\infty \\
& \dot{b}_{n}=b_{n}\left(a_{n+1}-a_{n}\right), \quad \infty
\end{aligned}
$$

with initial conditions:

$$
a_{n}(0)=a_{n}^{0}, \quad b_{n}(0)=b_{n}^{0},
$$

such that $a_{n}^{0}$ and $b_{n}^{0}-1 / 2$ decay faster that polynomially at $\infty$ and $-\infty$.

We will assume here that there are no solitons. In other words the initial data are such that the underlying linear discrete Lax operator has no eigenvalues. The solitons will be added in a later study.

It turns out that for large times $t$ one can distinguish three different regions.

1. In the region $|n / t|<1-C / t^{2-\delta}$, where $C, \delta$ are any given positive constants, the lattice performs decaying oscillations of order $0\left(1 / t^{1 / 2}\right)$. More precisely,

$$
4 b_{n}^{2}-1=s_{n}-s_{n-1},
$$


where

$$
s_{n}=\left(\frac{\nu\left(z_{0}\right)}{2 t \sin \theta_{0}}\right)^{1 / 2} \cos \left(2 t\left(\theta_{0} \cos \theta_{0}-\sin \theta_{0}\right)-\nu\left(z_{0}\right) \log t-\varepsilon\right)+o\left(t^{-1 / 2}\right)
$$

uniformly. Here $\theta_{0}$ is defined by

$$
\cos \theta_{0}=n / t
$$

while $z_{0}=e^{i \theta_{0}}$ and

$$
\begin{aligned}
\varepsilon= & \left.3 \nu\left(z_{0}\right) \log \left(2 \sin \theta_{0}\right)+\arg \left(r\left(z_{0}\right)\right)+\Gamma(-i \nu)\right) \\
& +\frac{\pi}{4}+\frac{1}{\pi} \int_{-w_{0}}^{w_{0}} \log \left(\frac{1-|r(z(w))|^{2}}{1-\left|r\left(z_{0}\right)\right|^{2}}\right) \frac{d w}{w-w_{0}}, \\
\nu(z)= & (-1 / 2 \pi) \log \left(1-\left|r\left(e^{i \theta}\right)\right|^{2}\right) \\
z(w)= & \frac{i-w}{i+w}, \quad z\left(w_{0}\right)=z_{0},
\end{aligned}
$$

$r$ being the reflection coefficient for the scattering of the underlying discrete Lax operator. (Note that $r$ is defined on the unit circle.) A similar expression can be written down for $a_{n}$.

2. In the case $\mid r(1)<1$, in the regions $\left|1 \pm \frac{n}{t}\right|<\frac{c}{t^{2}}$ the solution can be expressed by (0.3) with:

$$
s_{n}=\frac{1}{2 t^{1 / 3}} u\left(2 n t^{-1 / 3}-2 t^{2 / 3}\right)+0\left(t^{-2 / 3}\right)
$$

where $u$ is a particular solution of the Painlevé PII equation depending on the parameter $r(1)$.

3. In the region $\left|\frac{n}{t}\right|>1$, both $a_{n}$ and $b_{n}-1$ are $0\left(t^{-1}\right)$ for any positive $l$; the effect of the local disturbance is not yet felt.

Remarks. 1. The case $|r(1)|=1$ requires more work, and will be considered in a later paper.

2. The parametrisation of the 2-dimensional manifold of the solutions of PII can be defined either in terms of their long-time asymptotics or through the RiemannHilbert problems appearing in the related inverse monodromy problem; see Chap. 6 or [I-N]. The function $u$ appearing in (0.6) is in fact one in the 1-parameter family of non-singular solutions of PII.

3. A conclusion of the above results is that the speed of propagation of signals is finite, to leading order. As will be evident from the proof, this is due to the compactness of the spectrum of the underlying Lax operator. On the contrary, propagation is not finite in equations like $\mathrm{KdV}$ for example, where the underlying spectrum extends to infinity.

4. In our analysis we consider only positive $n$; the case where $n \leq 0$ is similar. 


\subsection{Solution and Background of the Problem}

There are three constituents to the proof of the above result: the restatement of the inverse scattering problem as a Riemann-Hilbert matrix factorization problem, the reduction of the Riemann-Hilbert problem to the solution of a singular integral equation according to the method of Beals and Coifman [B-C], and the use of their formulas to transform the initial factorization problem to one with a constant jump matrix, which can then be solved explicitly. The model of this procedure is the analogous treatment of the mKdV equation by Deift and Zhou [D-Z].

(i) The inverse scattering problem for the discrete Lax operator can be restated as a "Riemann-Hilbert matrix factorization problem" on the Riemann sphere, with discontinuity along the unit circle $C$ (for a proof of this fact, as well as a rudimentary discussion of the relevant inverse scattering theory the reader is referred to the appendix). The statement of this problem is as follows: to find the analytic matrixvalued function on $\mathbb{C}-C$, which satisfies a certain jump condition on $C$, and whose value at infinity is the unit matrix $I$ :

$$
\begin{aligned}
& Q_{+}(z)=Q_{-}(z) u_{n, t}(z), \quad z \in C, \\
& Q(z) \rightarrow I, \quad \text { as } z \rightarrow \infty
\end{aligned}
$$

where

$$
u_{n, t}(z)=\left(\begin{array}{cc}
1-|r(z)|^{2} & -\bar{r}(z) z^{2 n} e^{-t\left(z-z^{-1}\right)} \\
r(z) z^{-2 n} e^{t\left(z-z^{-1}\right)} & 1
\end{array}\right)
$$

(here, $Q_{+}$and $Q_{-}$are the normal limits on the circle $C$ from inside and outside respectively.)

Remarks. 1. In the main body of this paper, as opposed to the appendix, $r(z)$ (or $r(\theta)$ ) denotes the reflection coefficient at time zero.

2. From now on, unless explicitly states otherwise. given a $2 \times 2$ matrix $A$ we denote

$$
A_{n, t}=B^{-1} A B \text {, }
$$

where

$$
B=\left(\begin{array}{cc}
z^{-n} e^{t\left(z-z^{-1}\right) / 2} & 0 \\
0 & z^{n} e^{-t\left(z-z^{-1}\right) / 2}
\end{array}\right) .
$$

If in particular we have:

$$
u=\left(\begin{array}{cc}
1-|r(z)|^{2} & -\bar{r}(z) \\
r(z) & 1
\end{array}\right)
$$

then $u_{n, t}$ is given by $(0.8)$.

Indeed, one can express $b_{n}(t)$ in terms of $Q(0)$. In fact, if, say

$$
Q(0)=\left(\begin{array}{ll}
\alpha_{n} & \beta_{n} \\
\gamma_{n} & \delta_{n}
\end{array}\right)
$$

we can obtain

$$
\prod_{k=-\infty}^{k=n-1}\left(2 b_{k}\right)^{2}=\left(1+\beta_{n}\right) / \alpha_{n}
$$

Hence we can recover $b_{n}$ by dividing.

(ii) A crucial observation is that $u_{n, t}$ admits an upper-lower factorization

$$
u_{n, t}=\left(\left(b_{-}\right)_{n, t}\right)^{-1}\left(b_{+}\right)_{n, t},
$$


where

$$
\left(b_{-}\right)_{n, t}=\left(\begin{array}{cc}
z^{-n} e^{t\left(z-z^{-1}\right) / 2} & -\bar{r}(z) z^{n} e^{-t\left(z-z^{-1}\right) / 2} \\
0 & z^{n} e^{-t\left(z-z^{-1}\right) / 2}
\end{array}\right),
$$

and

$$
\left(b_{+}\right)_{n, t}=\left(\begin{array}{cc}
z^{-n} e^{t\left(z-z^{-1}\right) / 2} & 0 \\
r(z) z^{-n} e^{t\left(z-z^{-1}\right) / 2} & z^{n} e^{-t\left(z-z^{-1}\right) / 2}
\end{array}\right) .
$$

Define the "Cauchy" operators $C_{0}, C_{+}$and $C_{-}$as follows:

$$
\begin{aligned}
& \text { for } z \notin C, \quad \text { let }\left(C_{0} f\right)(z)=(2 \pi i)^{-1} \int_{C} \frac{f(w) d w}{w-z} \text { and } \\
& \text { for } z \in C, \quad \text { let }\left(C_{ \pm} f\right)(z)=(2 \pi i)^{-1} \int_{C} \frac{f(w) d w}{w-z^{ \pm}}
\end{aligned}
$$

( \pm signifying as the limit of the integral from inside (or outside) the circle $C$ ). Also, let the operator $C_{w_{n, t}}$ be defined by

$$
C_{w_{n, t}} f=C_{+}\left(f\left(w_{-}\right)_{n, t}\right)+C_{-}\left(f\left(w_{+}\right)_{n, t}\right)
$$

for a $2 \times 2$ matrix valued $f$, where

$$
w_{+}=b_{+}-I \quad \text { and } \quad w_{-}=I-b_{-} .
$$

Let $\mu$ solve the singular integral equation

$$
\mu=I+C_{w_{n, t}} \mu .
$$

Then, it follows easily that $Q$ defined by the integral formulae

$$
\begin{aligned}
& Q_{+}=I+C_{+}\left(\mu w_{n, t}\right), \quad Q=I+C_{0}\left(\mu w_{n, t}\right) \quad \text { on } \mathbb{C}-C, \\
& Q_{-}=I+C_{-}\left(\mu w_{n, t}\right),
\end{aligned}
$$

where $w=w_{+}+w_{-}$and thus $w_{n, t}=\left(w_{+}\right)_{n, t}+\left(w_{-}\right)_{n, t}$, provides the unique solution of (0.7). In particular, we compute $Q(0)$ hence we can compute $b_{n}(t)$. Thus, we obtain the solution of the Toda lattice in terms of the operator $\left(I-C_{w_{n, t}}\right)^{-1}$, provided of course that $I-C_{w_{n, t}}$ is invertible. Invertibility is proved in Chap. 3.

We note that this procedure can be carried out for any reasonable contour $\Sigma$ in the place of $C$. For each $\Sigma$ (with appropriate specification of its + side and - side) there are corresponding operators $C_{w_{n, t}}, C_{+}, C_{-}$, and each problem of type $(0.7)$ corresponds to a formula of type (0.18).

(iii) We "deform" the factorization problem (0.7) into one that can be solved explicitly, in a series of steps each of which involves either an extension or a deletion of a part of the factorization contour, or a substitution of $w_{ \pm}$by approximate (for large times) matrices, or an appropriate conjugation. The choice of the contour extensions or deletions depends on the phase of the exponentials appearing in $b_{ \pm}$. For each step, the final factorization problem is an approximation to the initial one in the sense that the solution as given by the corresponding integral formula $(0.18)$ is close to the exact solution of $(0.7)$.

Eventually, after repeated applications of this procedure we end up with an Riemann-Hilbert problem on the circle, with a constant jump matrix $u_{n, t}$ (independent of $z$ ), which can be solved explicitly in terms of parabolic cyclinder functions. Thus we also have an estimate for $Q(0)$, hence $b_{n}(t)$. 
More specifically, our procedure consists of eight steps:

(a) We observe that formula (0.18) involves the phase $\Phi=t\left(z-z^{-1}\right) / 2-n \log z$ appearing in the off-diagonal terms of $u_{n, t}(z)$. It thus suggests that we consider the regions for which $\Phi$ has real part decaying as $t \rightarrow \infty$. We then have to identify the contours on which $\operatorname{Re} \Phi$ is 0 , negative, or positive. We distinguish between the case $n / t<1$, the case where $1-n / t$ is small and the case $n / t>1$ (corresponding to the three regions defined in Sect.0.1). The following two figures provide the analysis of the sign of $\operatorname{Re} \Phi$ in the cases $n / t<1$ and $n / t>1$. Here $z_{0}=e^{i \theta_{0}}$.

N.B. From now on, up to and including Chap. 5, we restrict our analysis to the region $n / t<1$. The other regions are considered in Chap. 6 .
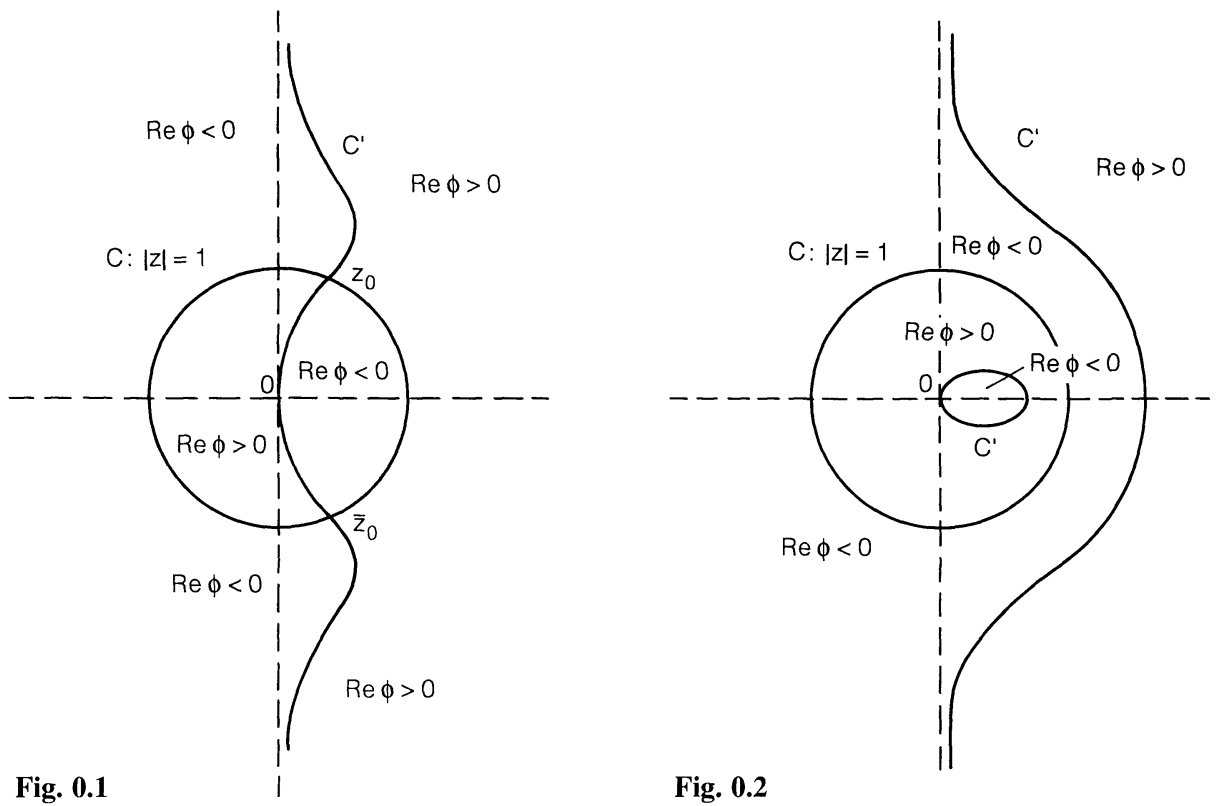

Fig. 0.2

Fig. 0.1. Analysis of the sign of $\operatorname{Re} \Phi$. Case I: $n / t<1$. The locus of $\operatorname{Re} \Phi=0$ consists of $C$ and $C^{\prime}$. Note: In this picture $n>0$; if $n<0$, the curve $C^{\prime}$ is on the left half-plane

Fig. 0.2. Analysis of the sign of $\operatorname{Re} \Phi$. Case II: $n / t>1$. Again the real part of the phase $\Phi$ is zero on the union of $C$ and $C^{\prime}$. When $n<0, C^{\prime}$ lies in the left half-plane

(b) Depending on the sign of $\operatorname{Re} \Phi$ we want an upper-lower or a lower-upper factorization for $u_{n, t}$. For $|\theta|>\theta_{0}$ [here $z=e^{i \theta}$ and $\theta_{0}$ is defined by (0.5)] our upperlower factorization is sufficient. For $|\theta|<\theta_{0}$ we need a lower-upper factorization. For this, we solve the scalar Riemann-Hilbert problem:

$$
\begin{aligned}
& d_{+}(z)=d_{-}(z)\left(1-|r(z)|^{2}\right) \text { for } z=e^{i \theta} \in C,|\theta|<\theta_{0}, \\
& d_{+}(z)=d_{-}(z) \text { for }|\theta|>\theta_{0}, \\
& d \rightarrow I \text { as } z \rightarrow \infty .
\end{aligned}
$$


which can be solved explicitly. Then, letting

$$
D(z)=\left(\begin{array}{cc}
d(z) & 0 \\
0 & (d(z))^{-1}
\end{array}\right)
$$

problem (0.7) is transformed into

$$
\begin{aligned}
& Q_{+}^{(1)}(z)=Q_{-}^{(1)}(z) u_{n, t}^{(1)}(z), \quad z \in C, \\
& Q^{(1)} \rightarrow I \quad \text { as } z \rightarrow \infty
\end{aligned}
$$

where $Q^{(1)}=Q D^{-1}$, and $u_{n, t}^{(1)}=D u_{n, t} D^{-1}$.

Now $u_{n, t}^{(1)}$ admits a lower-upper factorization for $|\theta|<\theta_{0}$ as shown:

$$
u_{n, t}^{(1)}=\left(\begin{array}{cc}
e^{-i \Phi} & 0 \\
0 & e^{i \Phi}
\end{array}\right)\left(\begin{array}{cc}
1 & 0 \\
-\bar{\varrho} & 0
\end{array}\right)\left(\begin{array}{ll}
1 & \varrho \\
0 & 1
\end{array}\right)\left(\begin{array}{cc}
e^{i \Phi} & 0 \\
0 & e^{-\imath \Phi}
\end{array}\right)
$$

where $\varrho=-\bar{r}(z)$ when $|\theta|>\theta_{0}$ and $\left.\varrho=-\bar{r}(z) /\left.(1-\mid r(z))\right|^{2}\right)$ when $|\theta|<\theta_{0}$.

(c) Using Fourier transforms we are able to split $\varrho$ into two parts, $\varrho=h_{1}+h_{2}$, s.t. $h_{1}$ decays as $t \rightarrow \infty$ and $h_{2}$ has an analytic continuation in the interior of the circle $C$. Similarly we have $-\varrho=h_{1}^{\prime}+h_{2}^{\prime}$, where $h_{1}^{\prime}$ decays as $t \rightarrow \infty$ and $h_{2}^{\prime}$ has an analytic continuation in the exterior of $C$.

By conjugating, we transform (0.21) into a problem of the form $(0.7)$ on the extended contour shown below (the choice of the contour is dictated by the situation in Figs. 0.1 and 0.2).

Fig. 0.3

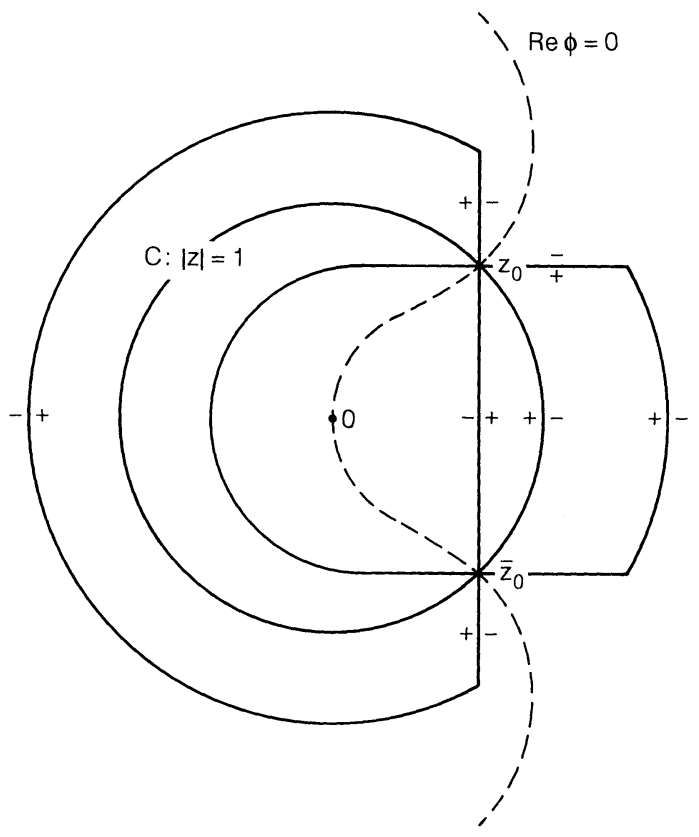

(d) On the circle $C$ the jump matrix depends on $h_{1}$ and is approximately $I$ for large times. This enables us to delete part (the circle $C$ ) of our contour. We now have a Riemann-Hilbert problem of type $(0.7)$ on the following contour: 
Fig. 0.4

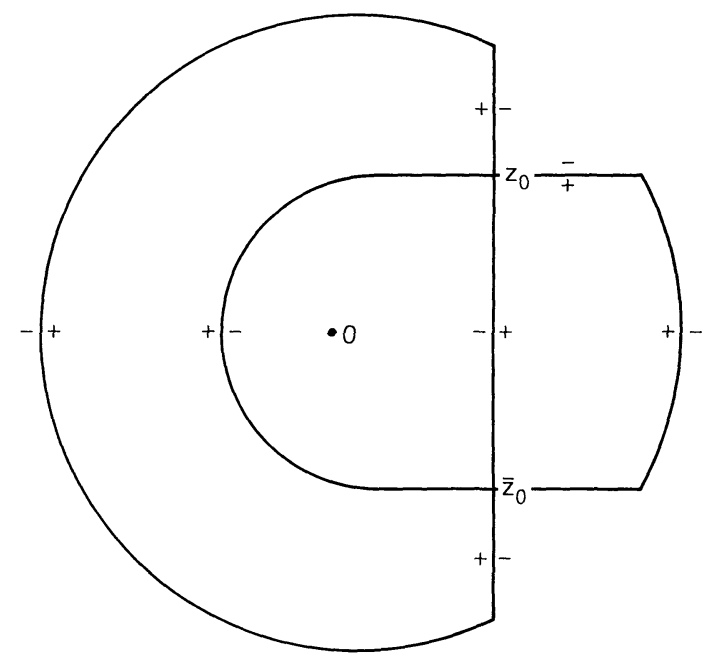

(e) Furthermore, if $\left|z-e^{i \theta_{0}}\right|$ is not small, the jump matrix $u_{n, t}$ has decaying offdiagonal entries. We are thus able to transform our factorization problem into one of type (0.7) on the contour consisting of two small crosses:

Fig. 0.5
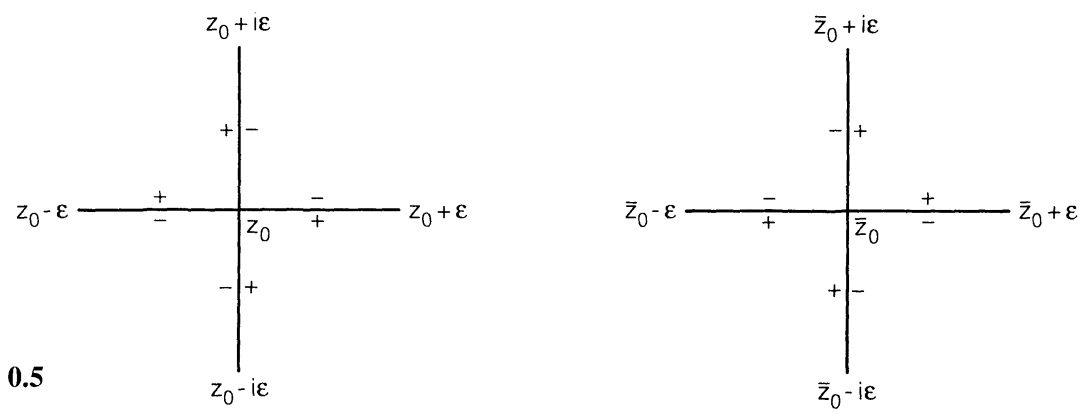

(f) We "decouple" the contribution of the two crosses. That is, we show that our answer for $Q$ can be obtained by solving each problem for each cross separately and adding each contribution.

(g) Through the scaling $\theta \rightarrow \theta\left(2 t \sin \theta_{0}\right)^{-1 / 2} \pm \theta_{0}$ each of the two problems is transformed to a problem on a small cross centered at $z=1$, of the type (0.7), but specific nontrivial asymptotics as $z \rightarrow \infty$.

(h) Finally, we transform the problem above to one on $C$ with constant jump matrix $u_{n, t}\left(e^{i \theta_{0}}\right)$. This final factorization problem can now be solved explicitly in terms of parabolic cylinder functions.

Steps (a) to (g) enable us to compute $Q(0)$ asymptotically for large times. Hence $b_{n}(t)$ is obtained. Similarly, one can obtain the asymptotics for $a_{n}(t)$.

\subsection{Background of the Problem}

The history of the treatment of long-time asymptotics for nonlinear integrable wave equations begins in the early 70's with the work of Zakharov and his collaborators. 
The first to include Riemann-Hilbert problems in his study of such asymptotics was A.R. Its (non-linear Schroedinger in $[\mathrm{I}], \mathrm{mKdV}$ in [I-N]), making use of the theory of isomonodromic deformations developed by the Japanese school (Miwa, Jimbo, Ueno etc.). The first fully rigorous treatment of such asymptotic problems involves Riemann-Hilbert factorization and was done recently by Deift and Zhou in [D-Z], in the context of the mKdV equation.

The corresponding asymptotic problem for a discrete integrable lattice was first treated by Novokshenov and Habibullin in 1981 (see [N-H]); explicit expressions were written down. Indeed, their recipe yields formulae answering our own problem. These formulae agree with ours except at a minor point: their formula for the last term in the phase, $\varepsilon$, is slightly different from ours. A more serious discrepancy is that they don't distinguish between the cases $|r(z=1)|=1$ and $|r(z=1)|<1$. However, the treatment (and indeed the behavior) of the problem is different in each case. Finally, it has to be said that the method of $[\mathrm{N}-\mathrm{H}]$ relies on the choice of the appropriate ansatz for the asymptotics. Our own method, on the contrary, is "algorithmic": no ansatz for the form of the asymptotic expressions is assumed.

\section{The Beals-Coifman Method}

We begin by restating the main result of the appendix.

Theorem 1.1 If $\left(a_{n}, b_{n}\right)$ solves the initial value problem $(0.1)-(0.2)$, then $b_{n}$ is given by

$$
\left(2 b_{n}\right)^{2}=\left(\frac{1+\beta_{n}}{\alpha_{n}}\right)^{1 / 2}\left(\frac{\alpha_{n-1}}{1+\beta_{n-1}}\right)^{1 / 2},
$$

where

$$
Q(z=0)=\left(\begin{array}{ll}
\alpha_{n} & \beta_{n} \\
\gamma_{n} & \delta_{n}
\end{array}\right) .
$$

Here $Q$ is the solution of the Riemann-Hilbert factorization problem

$$
\begin{aligned}
& Q_{+}=Q_{-} u_{n, t}(z), \\
& Q_{+} \rightarrow I, \quad \text { as } z \rightarrow \infty,
\end{aligned}
$$

where $u_{n, t}(z)$ is given by formula $(0.8)$.

The solution of (1.1) can be reduced to the solution of a singular integral equation as follows (see [B-C]). Consider an oriented contour $\Sigma$ on the complex plane, such that the number of its self-intersection points is finite. Define the matrix Cauchy operators as follows:

$$
\begin{gathered}
C_{ \pm}:\left(L^{2}(\Sigma)\right)_{2 \times 2} \rightarrow\left(L^{2}(\Sigma)\right)_{2 \times 2}, \\
\left.C_{ \pm} f\right)(z)=\frac{1}{2 \pi i} \int_{\Sigma} \frac{f(\zeta) d \zeta}{\zeta-z_{ \pm}},
\end{gathered}
$$

where $z \in \Sigma$ and the signs "+" and "-" connote normal limits as $z \rightarrow \Sigma$, from the right and the left of $\Sigma$ respectively (with respect to its orientation).

It is easy to see that $C_{+}$and $C_{-}$are bounded. It also follows easily from Cauchy's theorem that

$$
C_{+}-C_{-}=I .
$$


Next we define the matrix operator $C_{w}$ as follows: given any $2 \times 2$ matrices, say, $w_{+}$and $w_{-}$we define

$$
C_{w} f=C_{+}\left(f w_{-}\right)+C_{-}\left(f w_{+}\right) .
$$

Recall that

$$
u_{n, t}=\left(\left(b_{-}\right)_{n, t}\right)^{-1}\left(b_{+}\right)_{n, t},
$$

where $\left(b_{-}\right)_{n, t}$ and $\left(b_{+}\right)_{n, t}$ are given by formulae (0.11) and (0.12).

Let

$$
w_{+}=b_{+}-I, \quad w_{-}=I-b_{-},
$$

and define $\left(w_{+}\right)_{n, t}$ and $\left(w_{-}\right)_{n, t}$ according to (0.9). Also define

$$
w_{n, t}=\left(w_{+}\right)_{n, t}+\left(w_{-}\right)_{n, t},
$$

thus determining the corresponding operator $c_{w_{n, t}}$ accordingly. Suppose that $\mu(w ; n, t)$ solves the singular integral equation

$$
\mu=I+C_{w_{n, t}} \mu
$$

and define

$$
\begin{aligned}
Q(z) & =I+\frac{1}{2 \pi i} \int_{\Sigma} \frac{\mu(\zeta ; n, t) w_{n, t}(\zeta)}{\zeta-z} d \zeta, \\
Q_{+} & =I+C_{+}\left(\mu w_{n, t}\right), \\
Q_{-} & =I+C_{-}\left(\mu w_{n, t}\right) .
\end{aligned}
$$

In particular,

$$
Q(0)=I+\frac{1}{2 \pi i} \int_{\Sigma} \frac{\mu(\zeta ; n, t) w_{n, t}(\zeta)}{\zeta} d \zeta .
$$

Theorem 1.2. $Q, Q_{+}, Q_{-}$, as defined by (1.4) with $\Sigma=C$, provide the unique solution of the Riemann-Hilbert problem (1.1).

Proof. Existence follows by a simple straightforward calculation. For uniqueness suppose $Y_{1}$ and $Y_{2}$ are solutions of (1.1). Then $X=Y_{1}\left(X_{2}\right)^{-1}$ has no jump across $\Sigma$ hence it is entire and bounded, and thus constant by Liouville's theorem. By the condition at $\infty, Z=1$.

Remarks. 1. The procedure described above solves (1.1) provided $I-C_{w}$ in invertible. Existence of $\left(I-C_{w}\right)^{-1}$ will be proved in Chaps. 2-5.

2. Allowing solitons, i.e. allowing eigenvalues for $L$, is equivalent to allowing $Y$ to have poles. In that case existence of solutions to (1.1) is not always guaranteed and uniqueness holds only up to a choice of the residues at the poles. This case will be dealt with in a future paper.

Our problem is to be solved as follows. We begin with a problem of type (1.1) on the circle (that is $\Sigma=C$ ). Making use of estimates for $\left\|I-C_{w}\right\|$ we transform formula (1.4) to a similar one but on a different contour, up to a small error. (The BealsCoifman method applies unaltered to any reasonable contour on the Riemann Sphere.) Thus we reduce our Riemann-Hilbert problem above to one on a different contour. Repeating this procedure several times we eventually end up with a factorization problem on $\Sigma=C$ with a constant jump matrix, which can then be solved explicitly. Finally $b_{n}$ is recovered using Theorem 1.1 . 


\section{Contour Deformation}

I. We will first need to solve an auxiliary scalar factorization problem.

Let the "phase" $\Phi$ be defined by

$$
\Phi=\frac{1}{2} t\left(z-z^{-1}\right)-n \log z .
$$

The matrix $u_{n, t}$ can now be written as

$$
u_{n, t}=\left(\begin{array}{cc}
1-|r(z)|^{2} & -\bar{r}(z) e^{-2 i \Phi} \\
r(z) e^{2 \imath \Phi} & 1
\end{array}\right)=e^{-i \Phi \sigma_{3}} v e^{i \Phi \sigma_{3}} \equiv e^{i a d \Phi \sigma_{3}}
$$

where

$$
u=\left(\begin{array}{cc}
1-|r(z)|^{2} & -\bar{r}(z) \\
r(z) & 1
\end{array}\right) .
$$

Here, we have used the standard notation

$$
e^{a d x} A \equiv e^{-x} A e^{x},
$$

as well as the notation introduced by formulae $(0.9)-(0.10)$.

In the following Chaps. (2-5) we consider the region $0<n / t<1-C t^{-2+\delta}$, where $C$ is any positive constants (the other regions will be dealt with in Chap. 6). Thus the contour $\operatorname{Re} \Phi=0$ is as shown in Fig. 0.1. Let $z_{0}$ and $\bar{z}_{0}$ be the points of intersection of the unit circle $C$ and the curve $C^{\prime}$. We have $z_{0}=e^{i \theta_{0}}$ and $\bar{z}_{0}=e^{-\imath \theta_{0}}$, where $\cos \theta_{0}=n / t$. We also write $z=e^{i \theta}$ and from now on we are going to use variables $z$ and $\theta$ interchangeably; for example we may write $r(\theta)$ instead of $r(z)=r(z(\theta))$. When $z \in C, \theta$ is real; when $|z|<1, \operatorname{Im} \theta>0$ and when $|z|>1, \operatorname{Im} \theta<0$.

As long as $|\theta|>\theta_{0}$, we need an upper-lower factorization for $u$, in order to make good use of the results of Chap. 1. On the contrary, a lower-upper factorization will be necessary if $|\theta|<\theta_{0}$.

The lower-upper factorization is obvious:

$$
u=\left(\begin{array}{cc}
1 & -\bar{r}(z) \\
0 & 1
\end{array}\right)\left(\begin{array}{cc}
1 & 0 \\
r(z) & 1
\end{array}\right)
$$

and thus

$$
u_{n, t}=e^{-\imath a d \Phi \sigma_{3}}\left(\begin{array}{cc}
1 & -\bar{r}(z) \\
0 & 1
\end{array}\right)\left(\begin{array}{cc}
1 & 0 \\
r(z) & 1
\end{array}\right) .
$$

On the other hand, to produce an upper-lower factorization, we first need to consider the following scalar factorization problem on $C$.

$$
\begin{array}{rll}
d_{+}(z)=d_{-}(z)\left(1-|r(z)|^{2}\right), & \text { when }|\theta|<\theta_{0}, \quad d \rightarrow I, & \text { as } z \rightarrow \infty . \\
d_{+}(z)=d_{-}(z), & \text { when }|\theta|>\theta_{0}, &
\end{array}
$$

Such a problem can be solved explicitly. Indeed, consider the transformation

$$
w(z)=i \frac{1-z}{1+z}
$$

and let

$$
w_{0}=w\left(z_{0}\right)=i \frac{1-z_{0}}{1+z_{0}} .
$$


One finds

$$
\begin{aligned}
l(z)= & \exp \left(i \nu \log \left|\frac{w-w_{0}}{w+w_{0}}\right|-\nu \arg \left(w-w_{0}\right)+\nu \arg \left(w+w_{0}\right)\right) \\
& \exp \left(\frac{1}{2 \pi i} \int_{-w_{0}}^{w_{0}} \log \left(\frac{1-\left|r(z(\zeta))^{2}\right|}{1-\left|r\left(z_{0}\right)\right|^{2}}\right) \frac{d \zeta}{\zeta-w}\right) .
\end{aligned}
$$

Here

$$
\nu(z)=-\frac{1}{2 \pi} \log \left(1-|r(z)|^{2}\right)
$$

and

$$
\nu=\nu\left(z_{0}\right)
$$

We note that in the region $0<n / t<1-C t^{-2+\delta}$, $\sup _{z \in C}|r(z)|<\infty$; so $\nu$ is well-defined and positive, and

$$
\begin{array}{r}
\sup _{z \in C}|d(z)|<\infty, \\
\sup _{z \in C}\left|(d(z))^{-1}\right|<\infty .
\end{array}
$$

Let $D$ be the diagonal matrix given by

$$
D(z)=\left(\begin{array}{cc}
d(z) & 0 \\
0 & (d(z))^{-1}
\end{array}\right)
$$

and define

$$
Q^{(1)}=Q D^{-1}, \quad u^{(1)}=D_{-} u_{n, t} D_{+}^{-1}
$$

We then have the factorization problem on $C$,

$$
\begin{aligned}
Q_{+}^{(1)}=Q_{-}^{(1)} u^{(1)}, & \text { for } z \in C, \\
Q^{(1)} \rightarrow I, & \text { as } z \rightarrow \infty,
\end{aligned}
$$

where

$$
\begin{aligned}
u_{n, t}^{(1)}= & e^{-\imath a d \Phi \sigma_{3}}\left(\begin{array}{cc}
\frac{r(z)}{1-|r(z)|^{2}} d_{-}^{-2}(z) & 1
\end{array}\right)\left(\begin{array}{cc}
1 & \frac{-\bar{r}(z)}{1-|r(z)|^{2}} d_{+}^{2}(z) \\
0 & 1
\end{array}\right), \\
& \text { when }|\theta|<\theta_{0}, \\
u_{n, t}^{(1)}= & e^{-i a d \Phi \sigma_{3}}\left(\begin{array}{cc}
1 & -\bar{r}(z) d^{2}(z) \\
0 & 1
\end{array}\right)\left(\begin{array}{cc}
1 & 0 \\
r(z) d^{-2}(z) & 1
\end{array}\right), \\
& \text { when }|\theta|>\theta_{0} .
\end{aligned}
$$

For uniformity of notation we change the orientation of $C$ as follows. 


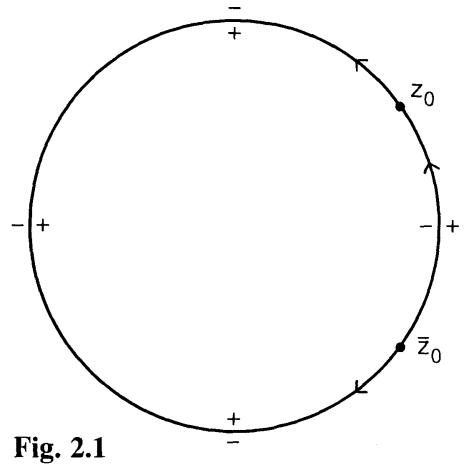

Fig. 2.1

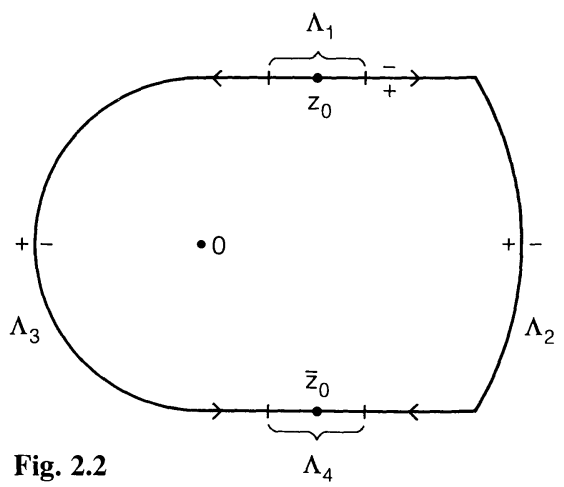

Remark. Giving an orientation to a contour is a handy way of distinguishing between its - side and its +side: the -side is to the "left" and the +side is to the "right". Thus, if the orientation of the unit circle is clockwise, the +side is to its interior while the - side is to its exterior, in agreement with the convention of Chap. 0. The labeling of - and + sides is necessary for the application of the Beals-Coifman method, as described in Chap. 1. In fact, the discussion of Chap. 1 goes through unaltered for any reasonable oriented contour (certainly for any of the contours introduced in this paper).

Thus our factorization problem is (2.15) with jump matrix given by (2.16), when $|\theta|<\theta_{0}$, and by

$$
u_{n, t}^{\left(1^{\prime}\right)}=e^{-i a d \Phi \sigma_{3}}\left(\begin{array}{cc}
1 & 0 \\
-r d^{-2} & 1
\end{array}\right)\left(\begin{array}{cc}
1 & \bar{r} d^{2} \\
0 & 1
\end{array}\right), \quad \text { when }|\theta|>\theta_{0} .
$$

II. As the reflection coefficient is not necessarily analytic in general, it will be convenient to split it as a sum of an analytic term and a term which is "small".

Consider the contour $\Lambda$ defined as follows (see Fig. 2.2).

$$
\Lambda=\Lambda_{1} \cup \Lambda_{2} \cup \Lambda_{3} \cup \Lambda_{4},
$$

where

$$
\Lambda_{1}=\left(z: z=z_{0}+s, s \in \mathbb{R},|s|<\varepsilon\right), \quad \Lambda_{4} \text { is the conjugate of } \Lambda_{1},
$$

while $\lambda_{2}$ and $\lambda_{3}$ are the circular arcs shown in Fig. 2.1 (the actual radii are immaterial as long as the geometry is as shown).

Furthermore, define

$$
\Lambda_{\varepsilon}=\Lambda_{2} \cup \Lambda_{3}
$$

Following [D-Z], we have

Theorem 2.1. Let $\varrho$ be defined on the unit circle $C$ as follows:

$$
\begin{aligned}
\varrho(z)=\frac{-\bar{r}(z)}{1-|r(z)|^{2}}, & \text { if }|\theta|<\theta_{0}, \\
\varrho(z)=\bar{r}(z), & \text { if }|\theta|>\theta_{0} .
\end{aligned}
$$

Then, we can decompose

$$
\varrho(z)=h_{I}(z)+h_{I I}(z)+R(z)
$$


where $h_{I}$ is small in the sense that

$$
\left|e^{-2 \imath \Phi(z)} h_{I}(z)\right| \leq c t^{-l}, \text { for all positive } l, z \in C,
$$

while $h_{I I}(z)$ can be continued analytically to the exterior of $C$, and

$$
\left|e^{-2 \imath \Phi(z)} h_{I I}(z)\right| \leq c t^{-l}, \quad \text { for all positive } l, z \in \Lambda,
$$

and $R(z)$ is a rational function of $\cos \theta$, smooth to any given order at the stationary phase points $\theta_{0},-\theta_{0}$, and satisfying

$$
\left|e^{-2 i \Phi(z)} R(z)\right| \leq c e^{-K \varepsilon^{2} t \sin \theta_{0}}, \quad z \in \Lambda_{\varepsilon}
$$

here $c, K$ are positive constants and $\varepsilon$ is an arbitrarily small positive number.

Proof. We consider two distinct cases separately.

(i) Case $|\theta|<\theta_{0}$. Recall that as we restrict ourselves to positive $n$, we have $0 \leq \theta_{0} \leq \pi / 2$. Expanding $\sigma(\cos \theta)$ using Taylor's theorem, we write:

$$
\begin{aligned}
\varrho(\cos \theta)= & \mu_{0}+\mu_{1}\left(\cos \theta-\cos \theta_{0}\right)+\ldots \mu_{k}\left(\cos \theta-\cos \theta_{0}\right)^{k} \\
& +\frac{1}{k !} \int_{\theta_{0}}^{\theta} \varrho^{(k+1)}(\gamma)(\cos \theta-\gamma)^{k} d \gamma .
\end{aligned}
$$

Let $R$ be the polynomial part of the right-hand side of (2.22), considered as a function of $\theta$. Then,

$$
\varrho=h+R,
$$

where

$$
\left.\frac{d^{j} h}{d \theta^{j}}\right|_{ \pm \theta_{0}}=0, \quad \text { for } 0 \leq j \leq K
$$

Next, we split

$$
h=h_{I}+h_{I I}
$$

with the required properties. Let

$$
\Theta=\frac{z-z^{-1}}{2}-\frac{n}{t} \log z
$$

so that $\Phi=t \Theta$, and

$$
\Theta=i\left(\sin \theta-\theta \cos \theta_{0}\right) \text { for } z=e^{i \theta} \in C .
$$

Let

$$
\alpha(\theta)=\left(\cos \theta-\cos \theta_{0}\right)^{q},
$$

and define

$$
\begin{array}{r}
h^{\alpha}(\Theta) \equiv \frac{h(\theta(\Theta))}{\alpha(\theta(\Theta))},|\Theta|<\Theta_{0}, \\
h^{\alpha}(\Theta) \equiv 0,|\Theta| \geq \Theta_{0},
\end{array}
$$

where $\Theta_{0}=\Theta\left(\theta_{0}\right)$. (Note that $\Theta(\theta)$ is invertible for $|\theta|<\theta_{0}$.) We define the Fourier transform

$$
\left(\widehat{h^{\alpha}}\right)(\eta)=\frac{1}{(2 \pi)^{1 / 2}} \int_{-\theta_{0}}^{\theta_{0}} e^{-i h \Theta(\theta)} h^{\alpha}(\theta) d \Theta
$$


and by the Fourier inversion theorem,

$$
h^{\alpha}(\Theta)=\frac{1}{(2 \pi)^{1 / 2}} \int_{-\infty}^{\infty} e^{i \eta \Theta(\theta)} \widehat{h^{\alpha}}(\eta), \quad \text { for }|\Theta|<\Theta_{0} .
$$

From the behavior of $h^{\alpha}$ near $\theta_{0}$ and $-\theta_{0}$ we can deduce that

$$
h^{\alpha}(\theta)=0\left(\theta^{2}-\theta_{0}^{2}\right)^{2(k+1-q)} .
$$

As

$$
\frac{d \theta}{d \Theta}=\frac{1}{\cos \theta-\cos \theta_{0}}
$$

we have

$$
\begin{aligned}
\int_{-\theta_{0}}^{\theta_{0}}\left|\left(\frac{d}{d \theta}\right)^{j} h^{\alpha}(\theta)\right|^{2}|d \theta| & =\int_{-\theta_{0}}^{\theta_{0}}\left|\left(\frac{1}{\cos \theta-\cos \theta_{0}} \frac{d}{d \theta}\right)^{j} h^{\alpha}\right|^{2}\left|\cos \theta-\cos \theta_{0}\right||d \theta| \\
& \leq \text { const. }<\infty, \quad \text { for } 0 \leq j \leq \frac{3 q+2}{2}
\end{aligned}
$$

Thus

$$
h^{\alpha} \in H^{j}(-\infty<\Theta<\infty), \quad \text { for } 0 \leq j \leq \frac{3 q+2}{2} .
$$

We now define $h_{I}$ and $h_{I I}$ as follows:

$$
\begin{aligned}
h_{I}(\theta) & =\frac{1}{(2 \pi)^{1 / 2}} \alpha(\theta) \int_{t}^{\infty} e^{i s \Theta(\theta)} \widehat{h^{\alpha}}(s) d s, \\
h_{I I}(\theta) & =\frac{1}{(2 \pi)^{1 / 2}} \alpha(\theta) \int_{-\infty}^{t} e^{i s \Theta(\theta)} \widehat{h^{\alpha}}(s) d s .
\end{aligned}
$$

Note that (2.25) is satisfied. Also,

$$
\begin{aligned}
\left|e^{2 i t \Theta(\theta)} h_{I}(\theta)\right| & \leq|\alpha(\theta)| \frac{1}{(2 \pi)^{1 / 2}} \int_{t}^{\infty}\left|\widehat{h^{\alpha}}(s)\right| d s \\
& \leq|\alpha(\theta)| \frac{1}{(2 \pi)^{1 / 2}}\left(\int_{t}^{\infty}\left(1+s^{2}\right)^{-p} d s\right)^{1 / 2}\left(\int_{t}^{\infty}\left(1+s^{2}\right)^{p}\left|\widehat{h^{\alpha}}(s)\right|^{2} d s\right)^{1 / 2} \\
& \leq c t^{p-1 / 2}, \quad \text { for } p \leq \frac{3 q+2}{2} .
\end{aligned}
$$

Clearly $h_{I I}$ can be continued analytically to the interior of $C$, and a similar argument to the above shows that indeed (2.21b) holds with $l=q / 2$; here, the crucial fact is that $\operatorname{Re}(i \theta)>0$ inside $C$. Finally, it is easy to see that $(2.21 \mathrm{c})$ holds on $\Lambda_{2}$.

(ii) Case $|\theta|>\theta_{0}$. The proof is similar. Now $i \Theta$ is positive in the exterior of $C$, so $h_{I I}$ can be extended analytically there (and is also small in the appropriate sense). 
Fig. 2.2

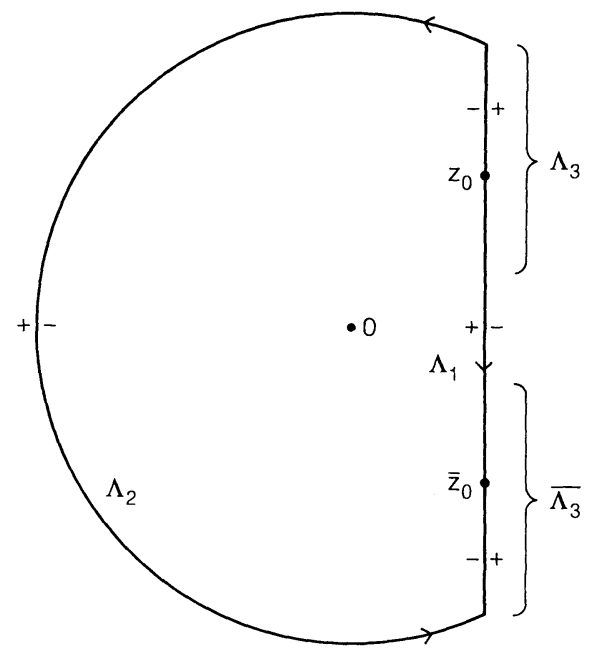

$I I^{\prime}$. Next, consider the contour $\Lambda^{\prime}$ as shown in Fig. $2.2^{\prime}$.

We have

$$
\Lambda^{\prime}=\Lambda_{1}^{\prime} \cup \Lambda^{\prime 2}
$$

where

$$
\Lambda_{1}^{\prime}=\left(z: z=\operatorname{Re} z_{0}+i s, s \in \mathbb{R},|s|<\operatorname{Im} z_{0}+\varepsilon\right)
$$

and $\Lambda_{2}^{\prime}$ is the circular arc shown (again the actual radius is immaterial.

Here,

$$
\Lambda_{\varepsilon}^{\prime}=\Lambda_{3}^{\prime} \cup \bar{\Lambda}_{3}^{\prime}, \quad \text { where } \quad \Lambda_{3}=\left(z: z=\operatorname{Re} z_{0}+i s, s \in \mathbb{R},|s|<\varepsilon\right) .
$$

Again we have a theorem analogous to Theorem 2.1, which enables us to split

$$
-\bar{\varrho}=h_{I}^{\prime}+h_{I I}^{\prime \prime}+R^{\prime}
$$

( $\varrho$ being defined by (2.19)): estimates (2.21a), (2.21b), and (2.21c) hold with $h$ replaced by $h^{\prime}$ and $\Lambda$ replaced by $\Lambda^{\prime}$.

III. We now augment our contour as indicated in Fig. 0.3. Let

$$
\Sigma^{(3)}=C \cup \Lambda \cup \Lambda^{\prime} \text {. }
$$

We rewrite the jump matrix (2.16)-(2.16) of the factorization problem (2.15) as follows:

$$
u_{n, t}^{(n)}=\left(b_{-}\right)_{n, t}^{-1}\left(b_{+}\right)_{n, t}
$$

with

$$
\left(b_{+}\right)_{n, t}=\left(\begin{array}{cc}
d & 0 \\
0 & d^{-1}
\end{array}\right) e^{-i \Phi} b_{+} e^{i \Phi}\left(\begin{array}{cc}
d^{-1} & 0 \\
0 & d
\end{array}\right),
$$

and similarly (in the notation introduced after (2.3) and using (2.13))

$$
\left(b_{-}\right)_{n, t}=D^{-1} e^{-i a d \Phi \sigma_{3}} b_{-} D \text {, }
$$


Fig. 2.3

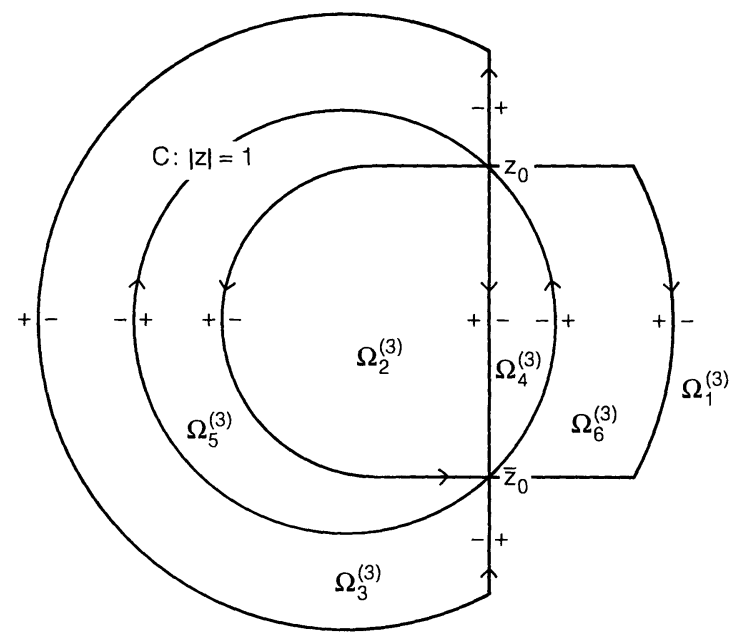

while (recall once more the notation of $(0.9)-(0.10)$ )

$$
b_{+}=\left(\begin{array}{ll}
1 & \varrho \\
0 & 1
\end{array}\right), \quad b_{-}=\left(\begin{array}{ll}
1 & 0 \\
\varrho & 0
\end{array}\right) .
$$

We split $b_{+}$and $b_{-}$according to the decomposition of $\varrho$ in (2.20); we define

$$
b_{+}^{0}=\left(\begin{array}{cc}
1 & h_{I} \\
0 & 1
\end{array}\right), \quad b_{+}^{a}=\left(\begin{array}{cc}
1 & h_{I I}+R \\
0 & 1
\end{array}\right)
$$

and similarly for $b_{-}^{0}, b_{-}^{a}$ in terms of $h_{I}^{\prime}, h_{I I}^{\prime}, R^{\prime}$.

We also write

$$
b_{+}=I+w_{+}, \quad b_{-}=I-w_{-},
$$

and similarly we introduce $w_{+}^{0}, w_{-}^{0}, w_{+}^{a}, w_{-}^{a}$.

Defining $Q^{(3)}$ on $\mathbb{C}-\Sigma^{(3)}$ (see Fig. 2.3) by

$$
\begin{aligned}
& Q^{(3)}(z)=Q(z)(D(z))^{-1}, z \in \Omega_{1}^{(3)} \cup \Omega_{2}^{(3)}, \\
& Q^{(3)}(z)=Q(z)(D(z))^{-1}\left(b_{-}^{a}\right)_{n, t}^{-1}, z \in \Omega_{3}^{(3)} \cup \Omega_{4}^{(3)}, \\
& Q^{(3)}(z)=Q(z)(D(z))^{-1}\left(b_{+}^{a}\right)_{n, t}^{-1}, z \in \Omega_{5}^{(3)} \cup \Omega_{6}^{(3)},
\end{aligned}
$$

we end up (after straightforward calculations) with a Riemann-Hilbert problem on $\Sigma^{(3)}$ as follows:

$$
Q_{+}^{(3)}(z)=Q_{-}^{(3)}(z) u_{n, t}^{(3)}(z), \quad \text { for } z \in \Sigma^{(3)}, Q^{(3)}(\infty)=I
$$

where

$$
\left.u_{n, t}^{(3)}(z)=\left(b_{-}^{(3)}\right)\right)_{n, t}^{-1}(z)\left(b_{+}^{(3)}\right)_{n, t}(z)
$$

and

$$
\begin{array}{ll}
b_{-}^{(3)}=b_{-}^{0}, z \in C, & b_{-}^{(3)}=I, z \in \Lambda, \\
b_{-}^{(3)}=b_{-}^{a}, z \in \Lambda^{\prime}, & b_{+}^{(3)}=b_{+}^{0}, z \in C, \\
b_{+}^{(3)}=b_{+}^{a}, z \in \Lambda, & b_{+}^{(3)}=I, z \in \Lambda^{\prime}
\end{array}
$$




\section{Reduction to a Problem on Two Crosses}

I. The main conclusion of the discussion in Chap. 2 is the following theorem.

Theorem 3.1. Let $\Sigma=C \cup \Lambda \cup \Lambda^{\prime}$ be the contour shown in Fig.0.3, and $\Sigma^{\prime}=$ $\Sigma-\left(C \cup \Lambda_{\varepsilon} \cup \Lambda_{\varepsilon}^{\prime}\right)$ be the union of the two crosses shown in Fig.3.1.

Fig. 3.1
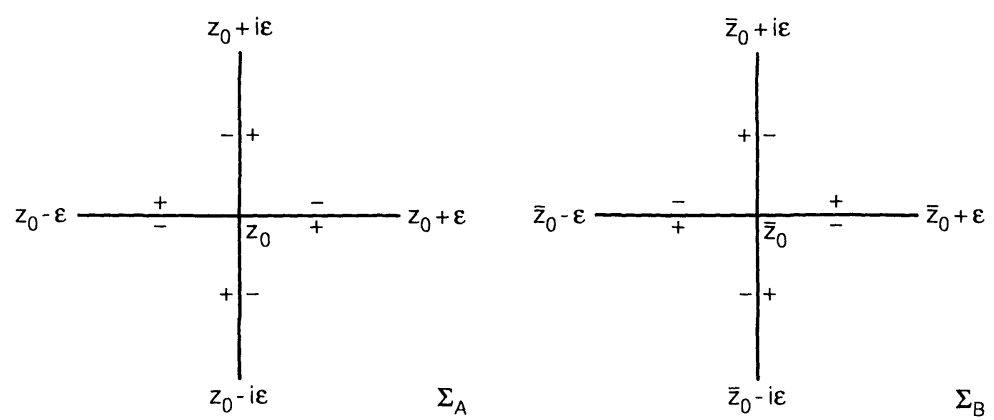

Recall that $w_{+}^{a}=b_{+}^{a}-I$ and $w_{-}^{a}=I-b_{-}^{a}$ (see discussion after in (2.44) in Chap. 2) and define

$$
w_{n, t}^{(3)}=\left(w_{+}^{(3)}\right)_{n, t}+\left(w_{-}^{(3)}\right)_{n, t}
$$

Then, we can write

$$
w_{n, t}^{(3)}=w^{\prime}+w^{e},
$$

where

$$
w^{\prime}=0, \quad \text { on } \Sigma-\Sigma^{\prime}
$$

and $w^{e}$ can be written as the sum

$$
w^{e}=w^{a}+w^{b}+w^{c}
$$

where

$w^{a}$ is supported on $C$ and is a sum of terms of types $h_{I}$ and $h_{I}^{\prime}$,

$w^{b}$ is supported on $\Lambda \cup \Lambda^{\prime}$ and is a sum of terms of types $h_{I I}$ and $h_{I I}^{\prime}$, $w^{c}$ is supported on $\Lambda_{\varepsilon} \cup \Lambda_{\varepsilon}^{\prime}$ and is a sum of terms of types $R$ and $\bar{R}$

Furthermore,

$$
\begin{aligned}
\left\|w^{a}\right\|_{L^{\infty} \cap L^{2} \cap L^{1}(C)} & \leq c t^{-l}, \\
\left\|w^{b}\right\|_{L^{\infty} \cap L^{2} \cap L^{1}\left(\Lambda \cup \Lambda^{\prime}\right)} & \leq c t^{-l}, \\
\left\|w^{c}\right\|_{L^{\infty} \cap L^{2} \cap L^{1}\left(\Lambda_{\varepsilon} \cup \Lambda_{\varepsilon}^{\prime}\right)} & \leq C e^{-K \varepsilon^{2} t \sin \theta_{0}}
\end{aligned}
$$

for all positive constants $c, C, K, \varepsilon$ being an arbitrarily small positive constant, and

$$
\left\|w^{\prime}\right\|_{L^{2}(\Sigma)} \leq C, \quad\left\|w^{e}\right\|_{L^{2}(\Sigma)} \leq C, \quad\left\|w_{n, t}^{(3)}\right\|_{L^{2}(\Sigma)} \leq C .
$$

Proof. Follows immediately from the discussion at the end of Chap. 2 and the estimates (2.21) of Sect. 2.II together with the analogous estimates of Sect. 2.II'.

II. Next, we reduce our problem to the solution of a factorization problem on $\Sigma^{\prime}$. We do this by transforming formula (1.4) to one of the same type, but on a different contour. 
The following identity is easy to check.

$$
\begin{aligned}
\int_{\Sigma}\left(\left(I-C_{w_{n, t}^{(3)}}\right)^{-1}\right) w_{n, t}^{(3)} \frac{d \zeta}{2 \pi i \zeta}-\int_{\Sigma}\left(\left(I-C_{w^{\prime}}\right)^{-1} I\right) w^{\prime} \frac{d \zeta}{2 \pi i \zeta} \\
=\int_{\Sigma} w^{e} \frac{d \zeta}{2 \pi i \zeta}+\int_{\Sigma}\left(I-C_{w^{\prime}}\right)^{-1}\left(C_{w^{e}} I\right) w_{n, t}^{(3)} \frac{d \zeta}{2 \pi i \zeta} \\
\quad+\int_{\Sigma}\left(I-C_{w^{\prime}}\right)^{-1}\left(C_{w^{\prime}} I\right) w^{e} \frac{d \zeta}{2 \pi i \zeta} \\
\quad+\int_{\Sigma}\left(I-C_{w^{\prime}}\right)^{-1} C_{w^{e}}\left(I-C_{w_{n, t}^{(3)}}\right)^{-1}\left(C_{\left.w_{n, t}^{(3)} I\right)} w_{n, t}^{(3)} \frac{d \zeta}{2 \pi i \zeta}\right.
\end{aligned}
$$

The right-hand side is of order $t^{-l}$. In fact,

Lemma 3.2. Let $A_{1}, A_{2}, A_{3}, A_{4}$ be the integrals appearing in the right-hand side of (3.1). Then

$$
\left|A_{i}\right| \leq\left(t \sin \theta_{0}\right)^{-l}
$$

for all $l$ and all $i$.

Proof. Follows from the estimates in Theorem (3.1), together with the bounds

$$
\left\|\left(I-C_{w^{\prime}}\right)^{-1}\right\|_{L^{2}(\Sigma)} \leq C, \quad\left\|\left(I-C_{w_{n, t}^{(3)}}\right)^{-1}\right\|_{L^{2}(\Sigma)} \leq C .
$$

For example

$$
\begin{aligned}
|B| & \leq\left\|\left(I-C_{w^{\prime}}\right)^{-1}\right\|_{L^{2}(\Sigma)}\left\|C_{w^{e}} I\right\|_{L^{2}(\Sigma)}\left\|w_{n, t}^{(3)}\right\|_{L^{2}(\Sigma)} \\
& \leq c\left\|w^{e}\right\|_{L^{2}(\Sigma)}\left\|w_{n, t}^{(3)}\right\|_{L^{2}(\Sigma)} \leq c\left(z_{0}\right) t^{-l},
\end{aligned}
$$

by the estimates (3.5) and (3.6).

A straightforward conclusion of the above and formula (1.5) is the following proposition.

Proposition 3.3. If $Q$ is the solution of the initial factorization problem (1.1), then

$$
Q^{3}(0)=Q(0) D(0)^{-2}=\int_{\Sigma}\left(\left(I-C_{w^{\prime}}\right)^{-1}\right)(\zeta) w^{\prime}(\zeta) \frac{d \zeta}{2 \pi i \zeta}+c\left(z_{0}\right)\left(t \sin \theta_{0}\right)^{-l},
$$

for any $l$.

We state the following result of [D-Z] without proof; it enables us to delete pieces of a contour on which the jump matrix is the identity.

Fact 3.4. Suppose we have two Riemann-Hilbert factorization problems $P$ and $P^{\prime}$, on contours $\Sigma$ and $\Sigma^{\prime}$ respectively, such that $\Sigma^{\prime}$ is a subset of $\Sigma$ and the jump matrix for $P$ on $\Sigma-\Sigma^{\prime}$ is the identity. Then $P$ and $P^{\prime}$ are equivalent; their solutions (as expressed by the Beals-Coifman formulae, for example) are equal.

The main result of this chapter now follows directly.

Theorem 3.5. If $\Sigma^{\prime}$ is as defined in Theorem 3.1,

$$
Q(0) D(0)^{-2}=\int_{\Sigma^{\prime}}\left(\left(I-C_{w^{\prime}}\right)^{-1} I\right)(\zeta) w^{\prime}(\zeta) \frac{d \zeta}{2 \pi i \zeta}+c\left(z_{0}\right)\left(t \sin \theta_{0}\right)^{-l},
$$

for any $l$. 
In other words (cf. Chap. 1), it suffices to solve the following Riemann-Hilbert problem on $\Sigma^{\prime}$ (up to an error $0\left(t^{-l}\right)$ ).

$$
Q_{+}^{(4)}(z)=Q_{-}^{(4)}(z) u_{n, t}^{(4)}(z), z \in \Sigma^{\prime}, \quad Q^{(4)}(\infty)=I,
$$

where

$$
u_{n, t}^{(4)}(z)=\left(\left(b_{-}^{(4)}\right)_{n, t}\right)^{-1}\left(b_{+}^{(4)}\right)_{n, t}
$$

and

$$
\begin{aligned}
& b_{+}^{(4)}=\left(\begin{array}{cc}
1 & R \\
0 & 1
\end{array}\right) \quad \text { on } \Lambda_{\varepsilon}^{\prime}, \\
& b_{+}^{(4)}=I \text { on } \Lambda_{\varepsilon}, \quad b_{-}^{(4)} I \text { on } \Lambda_{\varepsilon}^{\prime}, \\
& b_{-}^{(4)}=\left(\begin{array}{cc}
1 & 0 \\
R^{\prime} & 1
\end{array}\right) \text { on } \Lambda_{\varepsilon} .
\end{aligned}
$$

\section{Rescaling and Decoupling}

I. In this section, we decouple the contributions of the two crosses of Fig. 3.1, which constitute the contour $\Sigma^{(4)}=\Sigma_{A} \cup \Sigma_{B}$ as shown. Our procedure follows closely that of [D-Z] (Sect. 3).

Let $w^{(4)}=w_{A}+w_{B}$, where

$$
\begin{aligned}
& w_{A}=0, \quad \text { for } z \in \Sigma_{B}, \\
& w_{B}=0, \quad \text { for } z \in \Sigma_{A},
\end{aligned}
$$

Define $A=C_{w_{A}}$ and $B=C_{w_{B}}$ on $L^{1}\left(\Sigma^{(4)}\right)$ so that $C_{w}^{(4)}=A+B$.

Lemma 4.1. We have

$$
\|B A\|_{L^{2}\left(\Sigma^{(4)}\right)}=\|A B\|_{L^{2}\left(\Sigma^{(4)}\right)} \leq \frac{c\left(z_{0}\right)}{\left(t \sin \theta_{0}\right)^{1 / 2}}
$$

and

$$
\|A B\|_{L^{\infty}\left(\Sigma(4) \rightarrow L^{2}\left(\Sigma^{(4)}\right)\right.} \leq \frac{c\left(z_{0}\right)}{\left(t \sin \theta_{0}\right)^{1 / 2}}\left(z_{0} t\right)^{-1 / 4} .
$$

The proof is straightforward.

Lemma 4.2. $(I-A)^{-1}$ and $\left(I-B^{-1}\right.$ exist and are uniformly bounded.

Proof. Postponed until the end of Chap. 4 (Theorem 4.7).

Lemma 4.3. $\left(I-C_{\left.w^{(4)}\right)^{-1}}\right.$ exists and $\left\|\left(I-C_{w^{(4)}}\right)^{-1}\right\|_{L^{2}\left(\Sigma^{(4)}\right)} \leq C$.

Proof. Follows from Lemmas 4.1 and 4.2 (cf. [D-Z] Lemma 3.15) and the following identities:

$$
\begin{gathered}
(I-A-B)\left(I+A(I-A)^{-1}+B(I-B)^{-1}\right) \\
\quad=\left(I-B A(I-A)^{-1}-A B(I-B)^{-1}\right), \\
\left.I+A(I-A)^{-1}+B(I-B)^{-1}\right)(I-A-B) \\
=\left(I-(I-A)^{-1} A B-(I-B)^{-1} B A\right) .
\end{gathered}
$$

Lemma 4.4 $\left\|I-C_{w^{(4)}}\right\|_{\left.L^{2} \Sigma\right)}^{-1} \leq C$.

The proof is a consequence of Fact 3.4 (see [D-Z, Sect. 2]).

II. Next, we introduce a rescaling transformation for each cross.

First, we extend the contours $\Sigma_{A}$ and $\Sigma_{B}$ to the oriented contours $\hat{\Sigma}_{A}$ and $\hat{\Sigma}_{B}$ as shown. 


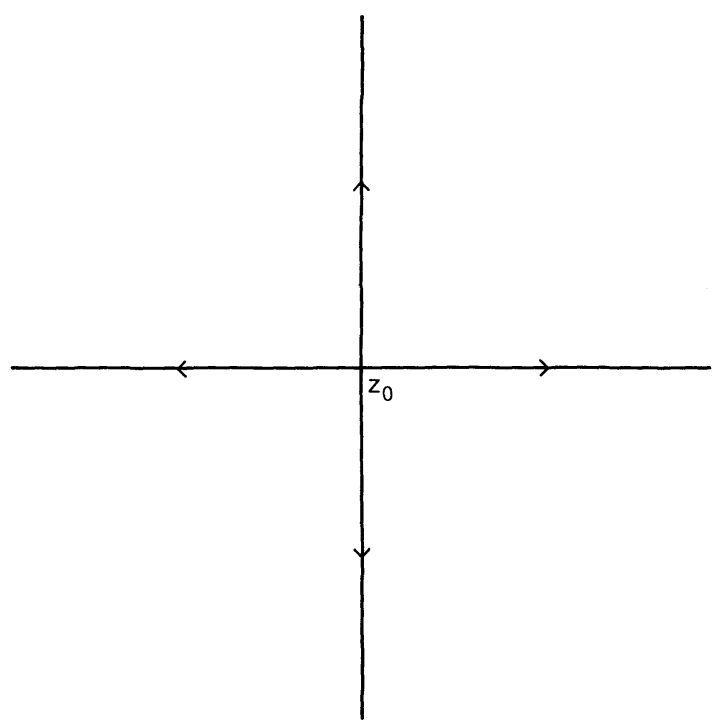

Fig. 4.1. Cross $\Sigma_{A}$

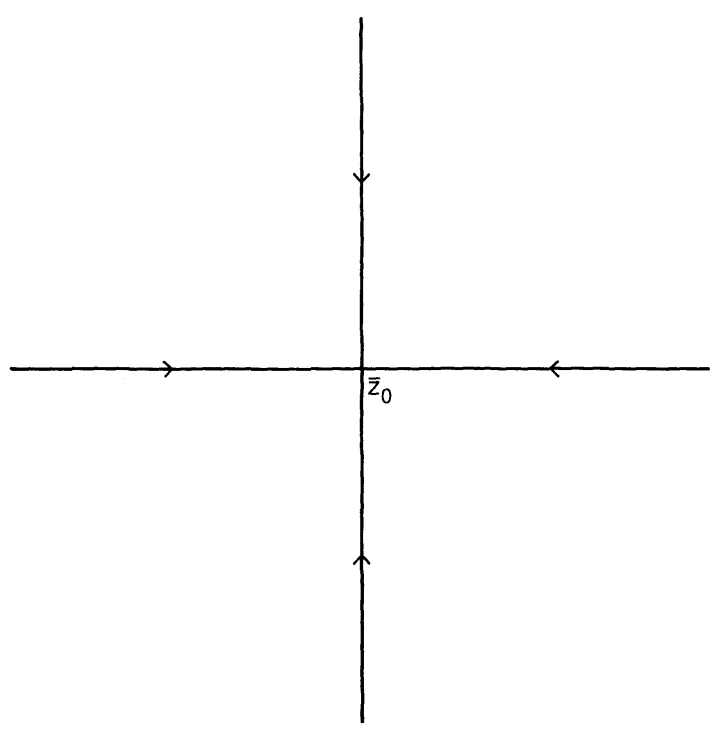

Fig. 4.2. Cross $\Sigma_{B}$

Let

$$
\hat{w}_{A}=w_{A}, z \in \Sigma_{A}, \quad \hat{w}_{A}=0, z \in \hat{\Sigma}_{A}-\Sigma_{A}
$$

and

$$
\hat{w}_{B}=w_{B}, z \in \Sigma_{B}, \quad \hat{w}_{B}=0, z \in \hat{\Sigma}_{B}-\Sigma_{A} .
$$

We introduce the scaling operators

$$
N_{A}: L^{2}\left(\hat{\Sigma}_{A}\right) \rightarrow L^{2}\left(\hat{\Sigma}_{A}\right), \quad N_{B}: L^{2}\left(\hat{\Sigma}_{B}\right) \rightarrow L^{2}\left(\hat{\Sigma}_{B}\right)
$$


by

$$
\begin{aligned}
& \left(N_{A} f\right)(z(\theta))=f\left(z\left(\frac{\theta}{\left(2 t \sin \theta_{0}\right)^{1 / 2}}-\theta_{0}\right)\right) \\
& \left(N_{B} f\right)(z(\theta))=f\left(z\left(\frac{\theta}{\left(2 t \sin \theta_{0}\right)^{1 / 2}}+\theta_{0}\right)\right)
\end{aligned}
$$

and we write

$$
\left(N_{B}\left(d e^{-i t \Theta}\right)\right)(z(\theta)) \equiv d_{B}^{0} d_{B}^{1}
$$

where

$$
d_{B}^{0} \equiv e^{i t\left(\cos \theta_{0}-\sin \theta_{0}\right)} t^{\frac{-\imath \nu}{2}}\left(2 \sin \theta_{0}\right)^{\frac{-3 i \nu}{2}} e^{i \chi}
$$

$\nu, \chi$ being defined by

$$
\begin{aligned}
\nu(\zeta) & =\frac{-1}{2 \pi} \log \left(1-|r(\zeta)|^{2}\right), \nu=\nu\left(z_{0}\right), \\
\chi & =-\frac{1}{2 \pi} \int_{-z_{0}}^{z_{0}} \log \left(\frac{1-|r(\zeta)|^{2}}{1-\left|r\left(z_{0}\right)\right|^{2}}\right) \frac{d \zeta}{\zeta-z_{0}} .
\end{aligned}
$$

Note that as long as $n / t>0,|r(\zeta)| \neq 1$, so $\nu(\zeta)>0$.

We have chosen factorisation (4.4) so that $d_{B}^{0}$ is independent of $z$ (or $\theta$ ). Note that all the factors in (4.5) are unitary except the last one. Thus

$$
\left|d_{B}^{0}\right|=1 \text {. }
$$

Let

$$
D_{B}^{0}=\left(\begin{array}{cc}
d_{B}^{0} & 0 \\
0 & \left(d_{B}^{0}\right)^{-1}
\end{array}\right)
$$

and $\tilde{D}_{B}^{0}$ denote right multiplication by $D_{B}^{0}$. We see that

$$
\hat{B}=\left(N_{B}\right)^{-1}\left(\tilde{D}_{B}^{0}\right)^{-1} B\left(\tilde{D}_{B}^{0}\right) N_{B},
$$

where

$$
B: L^{2}\left(\Sigma_{B}\right) \rightarrow L^{2}\left(\Sigma_{B}\right)
$$

is given by

$$
B=C_{\left(D_{B}^{0}\right)^{-1}\left(N_{B} \hat{w}_{B}\right) D_{B}^{0} .} .
$$

On the part $\Lambda_{B}^{\prime}=\Sigma_{B} \cap \Lambda^{\prime}$ of $\Lambda^{\prime}$, we have

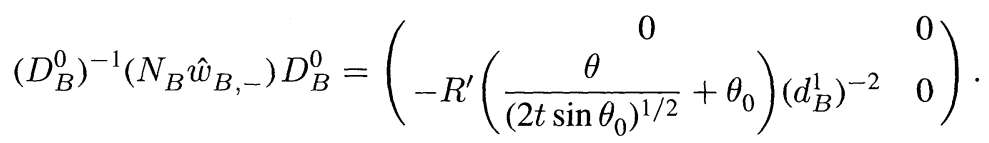

Otherwise

$$
\left(D_{B}^{0}\right)^{-1} N_{B} \hat{w}_{B,-} D_{B}^{0}=0 .
$$

On the part $\Lambda_{B}=\Sigma_{B} \cap \Lambda$ of $\Lambda$,

$$
\left.\left(D_{B}^{0}\right)^{-1}\left(N_{B} \hat{w}_{B,+}\right) D_{B}^{0}=\left(\begin{array}{cc}
0 & R\left(\frac{\theta}{\left(2 t \sin \theta_{0}\right)^{1 / 2}}+\theta_{0}\right. \\
0 & 0
\end{array}\right)\left(d_{B}^{1}\right)^{2}\right)
$$

and 0 otherwise.

By Lemmas (3.1)-(3.2), we have, after some calculations. 
Lemma 4.5. For $z(\theta) \in \Lambda_{B}$,

$$
\left.\left|R\left(\frac{\theta}{\left(2 t \sin \theta_{0}\right)^{1 / 2}}+\theta_{0}\right)\left(d_{B}^{1}\right)^{-2}-R\left(\theta_{0}+\right) \theta^{-2 \imath \nu} e^{\frac{2 \theta^{2}}{2}}\right| \leq c\left(z_{0}\right)\left|e^{i \gamma \theta^{2}}\right| t \sin \theta_{0}\right)^{-1 / 2}
$$

for any small enough $\gamma$, where

$$
\begin{aligned}
& R\left(\theta_{0}-\right) \equiv \lim _{\theta \uparrow \theta_{0}} R(\theta)=\frac{r\left(\theta_{0}\right)}{1-\left.r(\theta)\right|^{2}} \\
& R\left(\theta_{0}+\right) \equiv \lim _{\theta \downarrow \theta_{0}} R(\theta)=r\left(\theta_{0}\right) .
\end{aligned}
$$

Similarly, for $z(\theta) \in \Lambda_{B}^{\prime}$,

$$
\left|R^{\prime}\left(\frac{\theta}{\left(2 t \sin \theta_{0}\right)^{1 / 2}}+\theta_{0}\right)\left(d_{B}^{1}(\theta)\right)^{-2}-R^{\prime}\left(\theta_{0}+\right) \theta^{-2 i \nu} e^{\frac{i \theta^{2}}{2}}\right| \leq c\left(\theta_{0}\right) \mid e^{i \gamma \theta^{2}}\left(t \sin \theta_{0}\right)^{-1 / 2} \text {. }
$$

III. We can now use the above estimates to decouple the contributions due to the two crosses.

Theorem 4.6. If $Q$ is the solution of (3.12)-(3.14) (cf. Theorem 3.5), then

$$
\begin{aligned}
Q(0) D(0)^{-2}= & \int_{\Sigma_{A}}\left(I-C_{w_{A}}\right)^{-1} I(\zeta) w_{A}(\zeta) \frac{d \zeta}{2 \pi i \zeta}+\int_{\Sigma_{B}}\left(I-C_{w_{B}}\right)^{-1} I(\zeta) w_{B}(\zeta) \frac{d \zeta}{2 \pi i \zeta} \\
& +0\left(\frac{c\left(z_{0}\right)}{t\left(\theta_{0} \sin \theta_{0}\right)^{1 / 2}}\right) .
\end{aligned}
$$

Proof. Follows from the estimates above and the existence and boundedness of $(I-A)^{-1}$ and $(I-B)^{-1}$ (see Lemma 4.2 (cf. Proposition (3.66) of [D-Z]).

$I V$. We finally prove Lemma 4.2. Its proof is the result of the next step in our series of deformations.

Set

$$
w_{B}=\left(D_{B}^{0}\right)^{-1}\left(N_{B} \hat{w}\right) D_{B}^{0},
$$

so that $B=C_{w_{B}}$. On the cross $\Sigma_{B}$

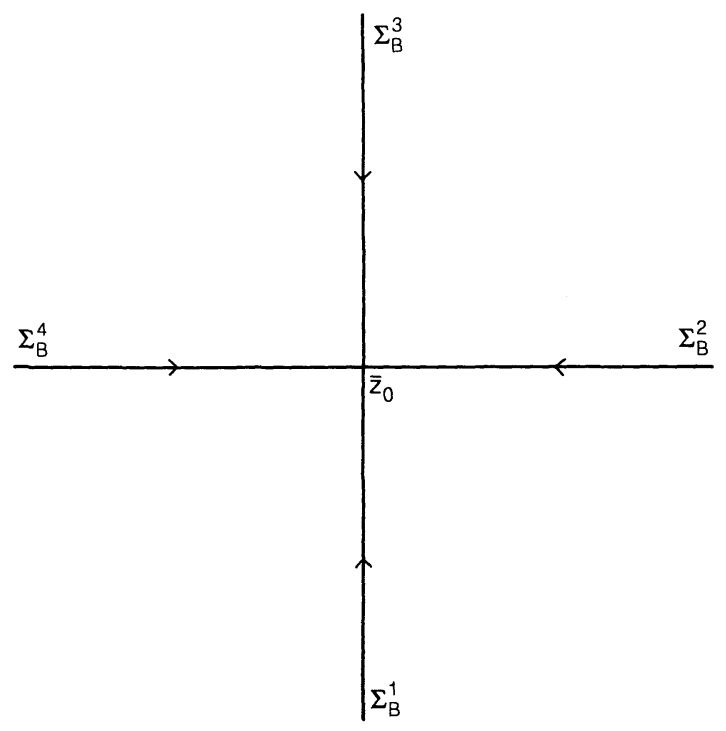

Fig. 4.3. Cross $\Sigma_{B}$ 
let

$$
v^{B^{0}}=\left(b_{-}^{B^{0}}\right)^{-1} b_{+}^{B^{0}},
$$

where

$$
b_{-}^{B^{0}}=I_{B}-w_{-}^{B^{0}}, \quad b_{+}^{B^{0}}=I_{B}-w_{+}^{B^{0}}
$$

and

$$
\begin{aligned}
& w^{B^{0}}=w_{+}^{B^{0}}(z)=\left(\begin{array}{ccc}
0 & \frac{-r\left(\theta_{0}\right)}{1-\left|r\left(\theta_{0}\right)\right|^{2}} \theta^{2 i \nu} e^{\frac{-i \theta^{2}}{2}} \\
0 & 0
\end{array}\right), z \in \Sigma_{B}^{1}, \\
& w^{B^{0}}=w_{-}^{B^{0}}(z)=\left(\begin{array}{cc}
\frac{\bar{r}\left(\theta_{0}\right)}{1-\left|r\left(\theta_{0}\right)\right|^{2}} \theta^{-2 \imath \nu} e^{\frac{\imath \theta^{2}}{2}} & 0
\end{array}\right), z \in \Sigma_{B}^{2}, \\
& w^{B^{0}}=w_{+}^{B^{0}}(z)=\left(\begin{array}{ccc}
0 & r\left(\theta_{0}\right) \theta^{2 \imath \nu} e^{\frac{-\imath \theta^{2}}{2}} \\
0 & 0 & 0
\end{array}\right), z \in \Sigma_{B}^{3}, \\
& w^{B^{0}}=w_{+}^{B^{0}}(z)=\left(\begin{array}{cc}
0 & 0 \\
-\bar{r}\left(\theta_{0}\right) \theta^{-2 i \nu} e^{\frac{i \theta^{2}}{2}} & 0
\end{array}\right), z \in \Sigma_{B}^{4},
\end{aligned}
$$

Let $B^{0}=C_{w^{B}}$. It is easy to prove the estimates

$$
\begin{aligned}
\left\|w_{B}-w^{B^{0}}\right\|_{L^{\infty} \cap L^{1} \cap L^{2}\left(\Sigma_{B}\right)} & \leq c\left(z_{0}\right)\left(t \sin \theta_{0}\right)^{-1 / 2} \\
\left\|B-B^{0}\right\|_{L^{2}\left(\Sigma_{A}\right)} & \leq c\left(z_{0}\right)\left(t \sin \theta_{0}\right)^{-1 / 2}
\end{aligned}
$$

It is now convenient to reorient $\Sigma_{B}$ as follows:

Fig. 4.4. $\Sigma_{B}$ (reoriented)

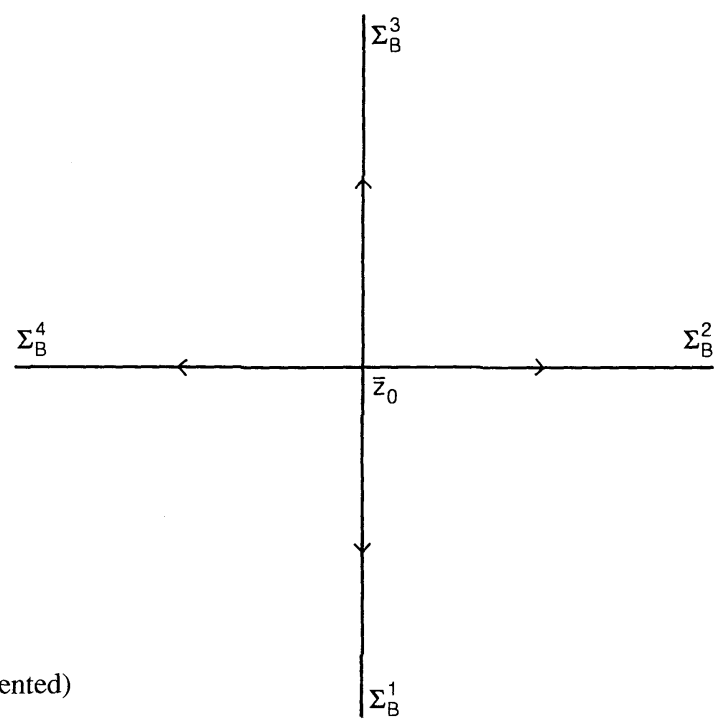

The resulting changes in the jump matrix are a change of sign for $w^{B^{0}}$ on $\Sigma_{B}^{2}$ and $\Sigma_{B}^{3}$. Next, we extend $\Sigma_{B}$ to $\Sigma_{B} \cup C=\tilde{\Sigma}_{B}$ with orientation as shown 


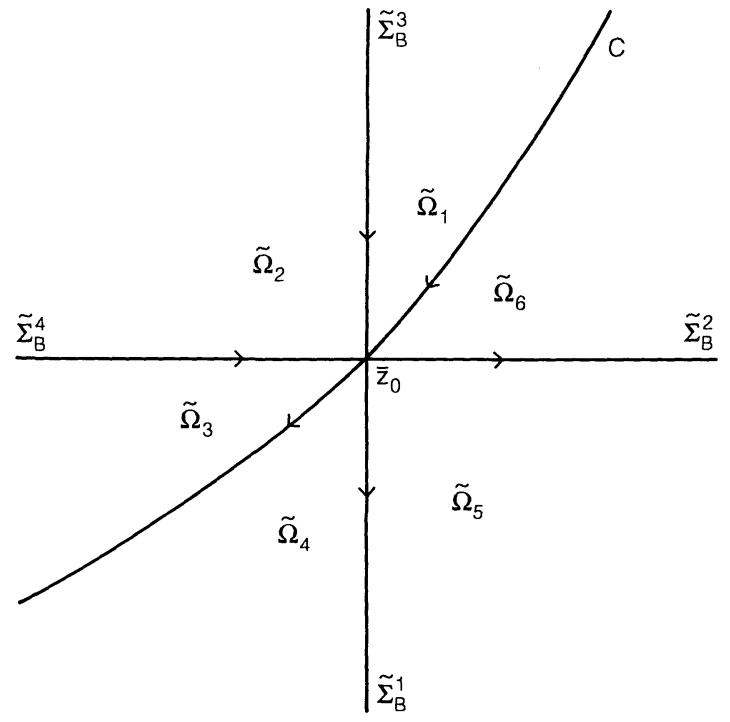

Fig. 4.5. $\tilde{\Sigma}_{B}$

and with

$$
\tilde{w}=w, z \in \Sigma_{B}, \quad \tilde{w}=0, z \in C .
$$

On $\tilde{\Sigma}_{B}$ we define a piecewise analytic function $\phi$ as follows:

$$
\begin{aligned}
& \phi(z)=(-\theta)^{i \nu \sigma_{3}}, z \in \tilde{\Omega}_{2} \cup \tilde{\Omega}_{5}, \\
& \phi(z)=(-\theta)^{i \nu \sigma_{3}}\left(b_{-}(z)\right)^{-1}, z \in \tilde{\Omega}_{1} \cup \tilde{\Omega}_{3}, \\
& \phi(z)=(-\theta)^{i \nu \sigma_{3}}\left(b_{+}(z)\right)^{-1}, z \in \tilde{\Omega}_{4} \cup \tilde{\Omega}_{6},
\end{aligned}
$$

Note that $b_{-}$and $b_{+}$can be continued analytically in $\tilde{\Omega}_{1} \cup \tilde{\Omega}_{3}$ and $\tilde{\Omega}_{4} \cup \tilde{\Omega}_{6}$ respectively. Next set

$$
\begin{aligned}
& \tilde{v}_{\phi}(z)=\phi_{-}(z) \tilde{v}(z)\left(\phi_{+}\right)^{-1}(z), z \in \tilde{\Sigma}-C, \\
& \tilde{v}_{\phi}(z)=I, z \in C .
\end{aligned}
$$

One checks that

$$
\tilde{v}_{\phi}(z)=e^{\frac{\imath \theta^{2}}{4}} v\left(-z_{0}\right) e^{\frac{-i \theta^{2}}{4}}, z \in C
$$

So

$$
\tilde{v}_{\phi}=\left(\tilde{b}_{-}\right)^{-1} \tilde{b}_{+}
$$

where

$$
\begin{aligned}
& \tilde{b}_{-}=\left(\begin{array}{ccc}
1 & e^{\frac{i \theta^{2}}{4}} \bar{r}\left(\bar{z}_{0}\right) \\
0 & 1
\end{array}\right), z \in C, \\
& \tilde{b}_{+}=\left(\begin{array}{ccc}
1 & 0 \\
e^{-\frac{-i \theta^{2}}{4}} r\left(\bar{z}_{0}\right) & 1
\end{array}\right), z \in C, \\
& \tilde{b}_{+}=I, z \in \tilde{\Sigma}-C .
\end{aligned}
$$

Finally, define

$$
H^{B^{0}}=Q^{B^{0}}(z)(\phi(z))^{-1}, z \in \mathbb{C}-\tilde{\Sigma}
$$


where $Q^{B^{0}}$ solves the Riemann-Hilbert problem corresponding to $\tilde{v}_{\phi}$, as defined above. One sees that there are no jumps across $\tilde{\Sigma}-C$. Thus $H(z)$ is analytic on $\mathbb{C}-C$ and

$$
H_{+}(z)=H_{-}(z) e^{\frac{-i \theta^{2}}{4} a d \sigma_{3}} v\left(z_{0}\right), z \in C \text {. }
$$

Also, as $z \rightarrow \infty$, we have

$$
H(z)=\theta^{i \nu \sigma_{3}}\left(I-\frac{Q_{1}^{B^{0}}}{z}+0\left(\frac{1}{z^{2}}\right)\right)
$$

and as $z \rightarrow 0$,

$$
H(z) \sim \theta^{\imath \nu \sigma_{3}}
$$

thus $H$ has essential singularities at 0 and $\infty$. Relations (4.11) and (4.12) constitute a singular Riemann-Hilbert problem on the circle which in fact can (and will, in Chap. 5) be solved explicitly.

Theorem 4.7. The operators $(I-A)^{-1},(I-B)^{-1},\left(I-C_{w^{\prime}}\right)^{-1},\left(I-C_{w^{A^{\prime}}}^{A^{\prime}}\right)^{-1}$ and $\left(I-C_{w^{B^{\prime}}}^{B^{\prime}}\right)^{-1}$ all exist and are bounded.

The proof is a direct consequence of the constructions above and depends on a series of subtle computations which enable one to factorise the operator $I-C_{\tilde{w}_{\phi}}$ as a product of two invertible operators. $\left(\tilde{w}_{\phi}\right.$ is defined in terms of the factors of $\tilde{v}_{\phi}$ in analogy with $w_{n, t}$ as defined in Chap. 1.) For details see Proposition 3.109 of [D-Z].

\section{A Second Order ODE and Parabolic Cylinder Functions}

I. In this chapter, we solve the factorization problem (4.11)-(4.12) explicitly. Recovering $H^{B^{0}}(z)$, we also obtain $Q^{B^{0}}(z)=H^{B^{0}}(z) \phi^{B^{0}}(z)$. Recalling that

$$
\begin{aligned}
Q_{+}^{B^{0}}(z) & =Q_{-}^{B^{0}}(z) u^{B^{0}}(z), z \in \Sigma_{B} \\
Q^{B^{0}}(\infty) & =I
\end{aligned}
$$

we have, by the Beals-Coifman formula,

$$
Q^{B^{0}}(z)=I+\int_{\Sigma_{B}}\left(I-B^{0}\right)^{-1}(\zeta) w^{B^{0}}(\zeta) \frac{d \zeta}{2 \pi i \zeta} .
$$

A simple calculation [using the rescaling definitions (4.3) and (4.8)] shows that

$$
\begin{aligned}
& \int_{\Sigma_{B}}\left(I-C_{w_{B}}\right)^{-1} I(\zeta) w_{B}(\zeta) \frac{d \zeta}{2 \pi i \zeta} \\
& \quad=\frac{1}{\left(2 t \sin \theta_{0}\right)^{1 / 2}} D_{B}^{0}\left(\int_{\Sigma_{B}}(I-B)^{-1} I(\zeta) w_{B}(\zeta) d \zeta\right)\left(D_{B}^{0}\right)^{-1} .
\end{aligned}
$$


Also, by (4.9) we have

$$
\begin{aligned}
\int_{\Sigma_{B}} & (I-B)^{-1} I(\zeta) w_{B}(\zeta) d \zeta \\
\quad= & \int_{\Sigma_{B}}\left(I-B^{0}\right)^{-1}(\zeta) w^{B^{0}}(\zeta) d \zeta+c\left(z_{0}\right)\left(t \sin \theta_{0}\right)^{-1 / 2} .
\end{aligned}
$$

Combining (5.1), (5.2), (5.3), (5.4) and using the analogous formulae for $Q^{A^{0}}$, we recover $Q(0)$ from Theorem (4.6) and hence $b_{n}(t)$ (see Chap. 1).

II. Define

$$
\Psi=H^{B^{0}} e^{-i \frac{\theta^{2}}{4} \sigma_{3}}
$$

Then

$$
\Psi_{+}(z)=\Psi_{-}(z) u\left(z_{0}\right) \text { on } C
$$

with

$$
\Psi \sim \theta^{i \nu \sigma_{3}} e^{-i \frac{\theta^{2}}{4} \sigma_{3}}, \quad \text { as } z(\theta) \rightarrow \infty
$$

Differentiating,

$$
\left(\frac{d \Psi}{d \theta}+\frac{1}{2} i \theta \sigma_{3} \Psi\right)_{+}=\left(\frac{d \Psi}{d \theta}+\frac{1}{2} i \theta \sigma_{3} \Psi\right)_{-} u\left(z_{0}\right)
$$

Thus, the function

$$
T=\left(\frac{d \Psi}{d \theta}+\frac{1}{2} i \theta \sigma_{3} \Psi\right) \Psi^{-1}
$$

has no jump across $C$, and noting that

$$
\Psi=\hat{\Psi} \theta^{i \nu \sigma_{3}} e^{-i \frac{\theta^{2}}{4} \sigma_{3}}
$$

where

$$
\hat{\Psi}=I+0(1 / z), \quad \text { near } z=\infty,
$$

we can see that $T$ is entire and bounded. It follows from Liouville's theorem that $T$ is constant. In fact, the value of the constant is extracted from the expansion of $H^{B^{0}}$ at infinity. Indeed,

$$
T=\left(\begin{array}{cc}
0 & \beta_{12} \\
\beta_{21} & 0
\end{array}\right)
$$

where

$$
\beta_{i j}=i\left(H_{1}^{B^{0}}\right)_{i j}
$$

with $H_{1}^{B^{0}}$ defined by

$$
H_{1}^{B^{0}}=\left(I-\frac{H_{1}^{B^{0}}}{z}+0\left(\frac{1}{z^{2}}\right)\right) \theta^{i \nu \sigma_{3}}, \quad \text { near } \infty,
$$

which defines $H_{1}^{B^{0}}$ once we keep in mind that $Q^{B^{0}}$, as defined in the beginning of this chapter, is analytic at $\infty$. 
We thus have

$$
\frac{d \Psi}{d \theta}+\frac{1}{2} i \theta \sigma_{3} \Psi=T \Psi
$$

Consider first the region $|z|>1$, i.e. $\operatorname{Im} \theta<0$. Letting $\Psi=\left(\Psi_{i j}\right)$, we obtain from (5.15),

$$
\frac{d^{2} \Psi_{11}}{d \theta^{2}}=\left(-\frac{\theta^{2}}{4}-\frac{i}{2}+\beta_{12} \beta_{21}\right) \Psi_{11} .
$$

Letting $\zeta=e^{3 i \pi / 4} \theta, a=i \beta_{12} \beta_{21}$ and $\Psi_{11}(\theta)=g(\zeta)$, we get

$$
\frac{d^{2} g}{d \zeta^{2}}+\left(\frac{1}{2}-\frac{\zeta^{2}}{4}+a\right) g(\zeta)=0 .
$$

This is the well-known parabolic cylinder equation, a set of linearly independent solutions for which is provided by $D_{a}(\zeta)$ and $D_{a}(-\zeta), D_{a}$ being the standard parabolic cylinder function.

Hence

$$
g(\zeta)=c_{1} D_{a}(\zeta)+c_{2} D_{a}(-\zeta)
$$

and thus

$$
\Psi_{11}(\theta)=c_{1} D_{a}\left(e^{\frac{-3 i \pi}{4}} \theta\right)+c_{2} D_{a}\left(-e^{\frac{-3 i \pi}{4}} \theta\right),
$$

for suitable constants $c_{1}, c_{2}$. Now, we know that, as $\operatorname{Im} \theta \rightarrow-\infty$, with $\operatorname{Re} \theta \in[-\pi, \pi]$, we have

$$
\begin{gathered}
D_{a}(\theta)=\theta^{a} e^{\frac{-\theta^{2}}{4}}\left(1+0\left(\theta^{-2}\right),\right. \\
D_{a}\left(e^{\frac{-32 \pi}{4}} \theta\right)=e^{\frac{-3 i \pi a}{4}} \theta^{a} e^{\frac{-i \theta^{2}}{4}}\left(1+0\left(\theta^{-2}\right)\right) .
\end{gathered}
$$

Also note that $H^{B^{0}}(\theta) \sim \theta^{i \nu \sigma_{3}}$, thus $\Psi_{11} \sim \theta^{i \nu} e^{\frac{-i \theta^{2}}{4}}$. Hence, $c_{2}=0, a=i \nu$, $c_{1}=e^{\frac{-3 \pi \nu}{4}}$, and

$$
\Psi_{11}(\theta)=e^{\frac{-3 \pi \nu}{4}} D_{i \nu}\left(e^{\frac{-3 i \pi}{4}} \theta\right), \text { for } \operatorname{Im} \theta<0 .
$$

In particular $\beta_{12} \beta_{21}=\varphi>0$. Similarity,

$$
\begin{aligned}
& \Psi_{12}(\theta)=\left(\beta_{21}\right)^{-1} e^{\frac{\pi \nu}{4}}\left(\frac{d}{d \theta}-\frac{i \theta}{2}\right) D_{-\imath \nu}\left(e^{\frac{-\imath \pi}{4}} \theta\right), \\
& \Psi_{21}(\theta)=\left(\beta_{12}\right)^{-1} e^{\frac{-3 \pi \nu}{4}}\left(\frac{d}{d \theta}+\frac{i \theta}{2}\right) D_{i \nu}\left(e^{\frac{-3 i \pi}{4}} \theta\right), \\
& \Psi_{22}(\theta)=e^{\frac{\pi \nu}{4}} D_{-i \nu}\left(e^{\frac{-\imath \pi}{4}} \theta\right) \text { for } \operatorname{Im} \theta<0 .
\end{aligned}
$$

When $|z|<1$, i.e. $\operatorname{Im} \theta>0$, we of course do not know the behavior of $H^{B^{0}}$ as $\operatorname{Im} \theta \rightarrow \infty$ a priori; it will be found by using the jump relation (5.6). We obtain

$$
\Psi_{11}(\theta)=d_{1} D_{\imath \nu}\left(e^{\frac{i \pi}{4}} \theta\right), \quad \Psi_{22}(\theta)=d_{2} D_{-i \nu}\left(e^{\frac{3 i \pi}{4}} \theta\right)
$$


and hence

$$
\begin{aligned}
& \Psi_{12}=\left(\beta_{21}\right)^{-1} d_{2}\left(\frac{d}{d \theta}-\frac{i \theta}{2}\right) D_{-i \nu}\left(e^{\frac{3 i \pi}{4}} \theta\right), \\
& \Psi_{21}=\left(\beta_{12}\right)^{-1} d_{1}\left(\frac{d}{d \theta}+\frac{i \theta}{2}\right) D_{i \nu}\left(e^{\frac{2 \pi}{4}} \theta\right) .
\end{aligned}
$$

Substituting in (5.6), we find

$$
\begin{aligned}
d_{1}=e^{\frac{\pi \nu}{4}}, & d_{2}=e^{\frac{-3 \pi \nu}{4}}, \\
\beta_{12}=\frac{(2 \pi)^{1 / 2} e^{\frac{\imath \pi}{4}} e^{\frac{-\pi \nu}{2}}}{r\left(z_{0}\right) \Gamma(-i \nu)}, & \beta_{21}=\frac{(2 \pi)^{1 / 2} e^{\frac{-i \pi}{4}} e^{\frac{-\pi \nu}{2}}}{\bar{r}\left(z_{0}\right) \Gamma(i \nu)} .
\end{aligned}
$$

We thus recover $\Psi(z=0)$ and by $(5.5) H^{B^{0}}$, hence $Q^{B^{0}}$. Indeed,

$$
Q^{B^{0}}(z=0)=I+\frac{i}{z}\left(\begin{array}{cc}
0 & \beta_{12} \\
\beta_{21} & 0
\end{array}\right)+0\left(z^{-2}\right) .
$$

Finally, using (5.2), (5.3), (5.4) and recalling formula (4.5) for $d_{0}^{B}$, we recover the contribution of the cross $\Sigma_{B}$. Analogous calculations show that is conjugate exactly equals the contribution from the cross $\Sigma_{A}$. We finally obtain (the asymptotic expression for) $Q(0)$,

$$
Q(0)=\left(2 t \sin \theta_{0}\right)^{1 / 2}\left(\begin{array}{cc}
1 & q_{n} \\
-q_{n} & 1
\end{array}\right)+0\left(\left(t \sin \theta_{0}\right)^{-1}\right)
$$

where

$$
q_{n}=\nu^{1 / 2} \cos \left(2 t\left(\theta_{0} \cos \theta_{0}-\sin \theta_{0}\right)-\nu \log t-\varepsilon\right), .
$$

where $\varepsilon$ is defined after formula (0.5). The final result is now immediate, once we recall formula (A.15) of the appendix, giving $b_{n}(t)$ in terms of $Q(t)$.

Remark. $D_{a}(\cdot)$ and $D_{a}(-\cdot)$ are always independent since $\beta_{12}$ and $\beta_{21}$ are clearly non-zero. Hence, the step leading to formula (5.18) is legitimate.

\section{The regions $\frac{n}{t} \sim 1$ and $\frac{n}{t}>1$}

I. We now consider the physically interesting region $\frac{C}{t^{2}}>\left|\frac{n}{t}-1\right|$. We distinguish between two cases $\frac{n}{t}<1$ and $\frac{n}{t}>1$, since the geometry of the contour $\operatorname{Re} \Phi=0$ is different in each case. We begin with the case where $\frac{n}{t}<1$.

In this case, the points $z_{0}$ and $\bar{z}_{0}$, defined by $z_{0}=e^{i \theta_{0}}, \cos \theta_{0}=\frac{n}{t}$ (see Fig. 2.1), are close to $z=1$.

Thus, we do not need a lower-upper factorization in the region $|\theta|<\theta_{0}$. In fact, we have (as before),

$$
u_{n, t}(z)=\left(\left(b_{-}\right)_{n, t}\right)^{-1}\left(b_{+}\right)_{n, t}=e^{-i t \Theta(z)}\left(\begin{array}{cc}
1 & -\bar{r}(z) \\
0 & 1
\end{array}\right)\left(\begin{array}{cc}
1 & 0 \\
r(z) & 1
\end{array}\right) e^{i t \Theta(z)}
$$


and we decompose

$$
\begin{aligned}
r(z) & =h_{I}(z)+h_{I I}(z)+R(z), \\
-\bar{r}(z) & =h_{I}^{\prime}(z)+h_{I I}^{\prime}(z)+R^{\prime}(z),
\end{aligned}
$$

as in Chap. 2. This time, however, we only extend our factorization problem to the contour shown in Fig. 6.1.

Fig. 6.1

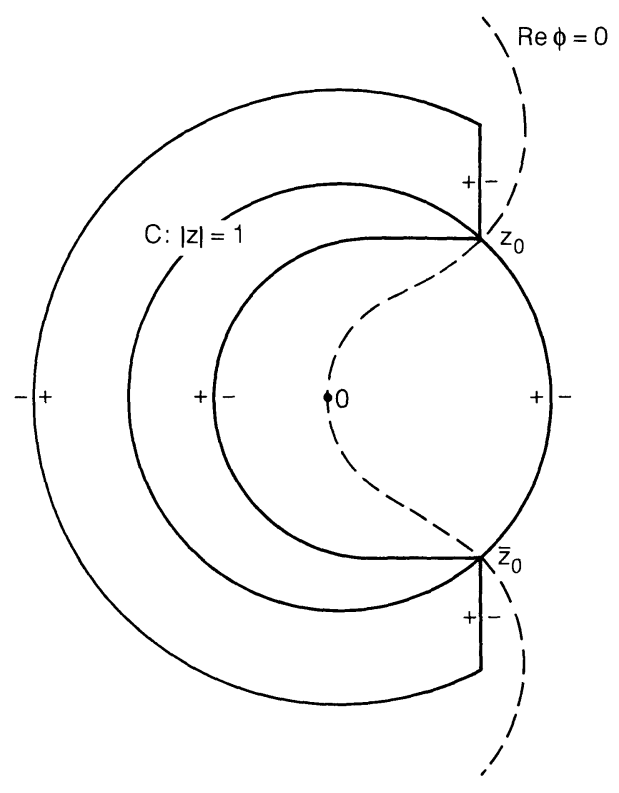

We now have (through a procedure analogous to that of Chap. 2)

$$
\begin{aligned}
u_{n, t}^{(1)}(z) & =\left(b_{+}\right)_{n, t} \\
& =e^{-i t \Theta(z) a d \sigma_{3}}\left(\begin{array}{cc}
1 & 0 \\
h_{I I}^{\prime}(z) & 1
\end{array}\right), z \in \Sigma_{3}^{(1)}, \\
u_{n, t}^{(1)}(z) & =\left(\left(b_{-}\right)_{n, t}\right)^{-1} \\
& =e^{-i t \Theta(z) a d \sigma_{3}}\left(\begin{array}{cc}
1 & -h_{I I}(z) \\
0 & 1
\end{array}\right), z \in \Sigma_{1}^{(1)}, \\
u_{n, t}^{(1)}(z) & =\left(\left(b_{-}\right)_{n, t}\right)^{-1}\left(b_{+}\right)_{n, t} \\
& =e^{-\imath t \Theta(z) a d \sigma_{3}}\left(\begin{array}{cc}
1-|r(z)|^{2} & -\bar{r}(z) \\
r(z) & 1
\end{array}\right), z \in \Sigma_{4}^{(1)}, \\
u_{n, t}^{(1)}(z) & =\left(\left(b_{-}\right)_{n, t}\right)^{-1}\left(b_{+}\right)_{n, t} \\
& =e^{-\imath t \Theta(z) a d \sigma_{3}}\left(\begin{array}{cc}
1 & -h_{I}(z)-R(z) \\
0 & 1
\end{array}\right)\left(\begin{array}{cc}
1 & 0 \\
h_{I}^{\prime}(z)+R^{\prime}(z) & 1
\end{array}\right), z \in \Sigma_{2}^{(1)},
\end{aligned}
$$

Again, contributions from $h_{I}, h_{I}^{\prime}, h_{I I}$ and $h_{I I}^{\prime}$ on $\Sigma_{1}^{(1)}$ and $\Sigma_{3}^{(1)}$ are of order $t^{-2}$. Thus we can delete pieces of $\Sigma^{(1)}$, ending up with a factorization problem on the unit circle $\Sigma^{(2)}$ as shown. 
Fig. 6.2. Contour $\Sigma^{(2)}$

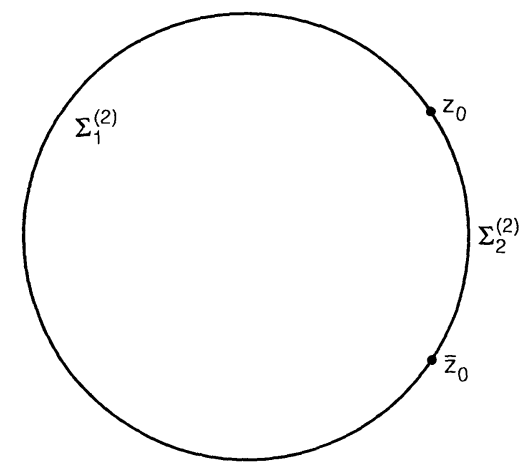

Here

$$
u_{n, t}^{(2)}=\left(\left(b_{-}^{(2)}\right)_{n, t}\right)^{-1}\left(b_{+}^{(2)}\right)_{n, t},
$$

where

$$
\left(b_{ \pm}^{(2)}\right)_{n, t}=\left(b_{ \pm}\right)_{n, t}, z \in \Sigma_{1}^{(2)}
$$

and

$$
\begin{aligned}
& \left(b_{-}^{(2)}\right)_{n, t}=e^{-i t \Theta(z) a d \sigma_{3}}\left(\begin{array}{cc}
1 & R(z) \\
0 & 1
\end{array}\right), z \in \Sigma_{2}^{(2)}, \\
& \left(b_{+}^{(2)}\right)_{n, t}=e^{-i t \Theta(z) a d \sigma_{3}}\left(\begin{array}{cc}
1 & 0 \\
R^{\prime}(z) & 1
\end{array}\right), z \in \Sigma_{2}^{(2)},
\end{aligned}
$$

with $R$ smooth to any desired order at $z_{0}$ and $\bar{z}_{0}$, and $R\left(e^{ \pm i \theta_{0}}\right)=r\left(e^{ \pm i \theta_{0}}\right)$.

At this point it will be convenient to restate our factorization problem in the $\theta$ plane, where $z=e^{i \theta}$. The inverse image of the unit circle is the real line and functions $r, \Theta, b_{ \pm}, u_{n, t}$ etc. will be considered as (periodic) functions of $\theta$ (with period $2 \pi$ ). The inverse images $\Sigma_{2}^{(3)}$ of $\Sigma_{2}^{(2)}$ and $\Sigma_{1}^{(3)}$ of $\Sigma_{1}^{(2)}$ are infinite unions of intervals.

$\begin{array}{llllllllll}-\theta_{0}-2 n & \theta_{0}-2 n & -2 \pi & -\theta_{0} & 0 & \theta_{0} & 2 \pi & -\theta_{0}+2 n & \theta_{0}+2 n \\ \Sigma_{2}^{(3)} & \Sigma_{1}^{(3)} & \Sigma_{2}^{(3)} & \Sigma_{1}^{(3)} & \Sigma_{2}^{(3)} & \Sigma_{1}^{(3)}\end{array}$

Fig. 6.3. Contour $\Sigma^{(3)}$ in the $\theta$-plane

We then have a factorization problem with jump matrix

$$
u_{n, t}^{(3)}=\left(\left(b_{-}^{(3)}\right)_{n, t}^{-1}\left(b_{+}^{(3)}\right)_{n, t},\right.
$$

with

$$
\begin{aligned}
& \left(b_{ \pm}^{(3)}\right)_{n, t}=\left(b_{ \pm}\right)_{n, t}, \theta \in \Sigma_{1}^{(3)}, \\
& \left(b_{-}^{(3)}\right)_{n, t}=e^{-\imath t \Theta(\theta) a d \sigma_{3}}\left(\begin{array}{cc}
1 & R(\theta) \\
0 & 1
\end{array}\right), \theta \in \Sigma_{2}^{(3)} \\
& \left(b_{+}^{(3)}\right)_{n, t}=e^{-\imath t \Theta(\theta) a d \sigma_{3}}\left(\begin{array}{cc}
1 & 0 \\
R^{\prime}(\theta) & 1
\end{array}\right), \theta \in \Sigma_{2}^{(3)}
\end{aligned}
$$

Rescaling

$$
\phi=2 t^{1 / 3} \theta,
$$

we obtain a new Riemann-Hilbert problem on the line (see Fig. 6.4). 


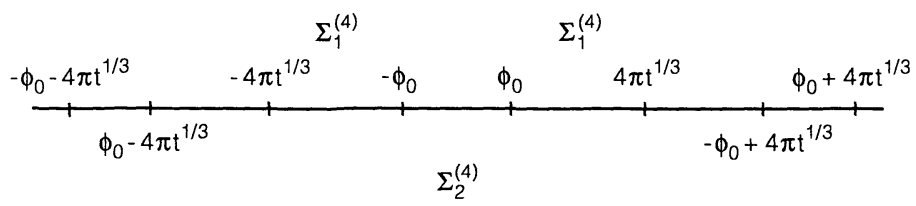

Fig. 6.4. Contour $\Sigma^{(4)}$

The set of stationary point is now the infinite discrete set $\left( \pm \phi_{0}+4 k \pi t^{1 / 3}, k \in \mathbb{Z}\right)$; the points $\pm \phi_{0}$ lie in a fixed bounded segment. Our jump matrix is $u_{n, t}^{(4)}(\phi)=u_{n, t}^{(3)}(\theta)$ and the condition $Q(\infty)=1$ becomes

$$
\lim _{\operatorname{Im} \theta \rightarrow-\infty} Q(\theta)=1 .
$$

We will next show that one can ignore the contributions of all stationary points but $\phi_{0}$ and $-\phi_{0}$.

Let

$$
\Phi(\phi) \equiv \frac{4}{3}\left(-\phi^{3}+2\left(t^{2 / 3}-n t^{-1 / 3}\right) \phi\right)
$$

and note that for large times

$$
t \Theta(\phi) \sim \frac{-t \theta^{3}}{6}+(t-n) \theta=\Phi(\phi)
$$

with uniform error $0\left(t^{-2 / 3}\right)$. Also note that

$$
\left(b_{+}^{(4)}\right)_{n, t}(\phi)=e^{-i \Phi a d \sigma_{3}} b_{+}(\phi=0)+0\left(t^{-2 / 3} e^{i \Phi}\right), \phi \in \Sigma_{2}^{(4)},
$$

and similarly for $\left.b_{-}^{(4)}\right)_{n, t}$. In fact for such $\phi$ the error is readily seen to be $0\left(t^{-2 / 3}\right)$.

Thus, the points of intersection of the curve $\operatorname{Re} \Phi=0$ and the unit circle are just $\phi_{0}$ and $-\phi_{0}$. Our solution can now be found in terms of the Riemann-Hilbert problem with

$$
u_{n, t}^{(4)}=e^{-\imath \Phi a d \sigma_{3}} u(\phi=0), \phi \in \Sigma_{2}^{(4)} .
$$

Now, $\Sigma^{(4)}$ being independent of $t$, we can delete a piece and augment as follows. Let $\Sigma^{(5)}$ be as shown in Fig. 6.5.

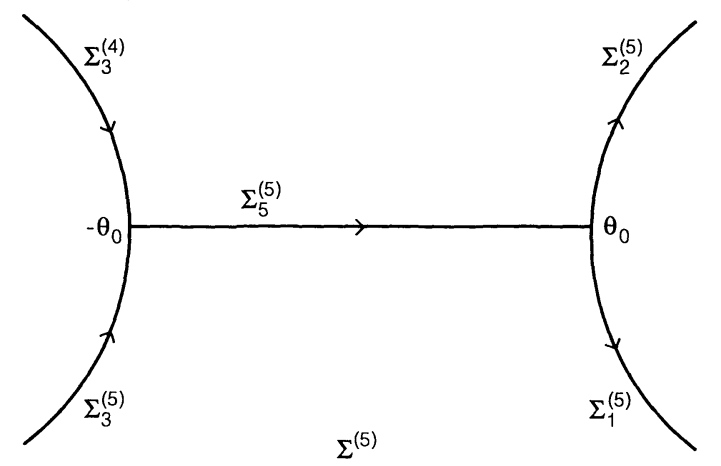

Fig. 6.5. Contour $\Sigma^{(5)}$ 
We have a factorization on $\Sigma^{(5)}$ with

$$
u_{n, t}^{(5)}(\phi)=u_{n, t}^{(4)}(\phi)=u_{n, t}^{(3)}(\phi), \phi \in \Sigma_{5}^{(5)}
$$

and

$$
u_{n, t}^{(5)}=\left(\left(b_{-}^{(5)}\right)_{n, t}\right)^{-1}\left(b_{+}^{(5)}\right)_{n, t},
$$

where

$$
\left(b_{-}^{(5)}\right)_{n, t}=e^{-i \Phi(\phi) a d \sigma_{3}}\left(\begin{array}{cc}
1 & 0 \\
R^{\prime}(\phi) & 1
\end{array}\right), \phi \in \Sigma_{2}^{(5)} \cup \Sigma_{3}^{(5)}
$$

and

$$
\left(b_{+}^{(5)}\right)_{n, t}=e^{i \Phi(\phi) a d \sigma_{3}}\left(\begin{array}{cc}
1 & R(\phi) \\
0 & 1
\end{array}\right), \phi \in \Sigma_{1}^{(5)} \cup \Sigma_{4}^{(5)} .
$$

The next step to augment the contour of Fig. 6.5 as shown in Fig. 6.6 and consider the following problem:

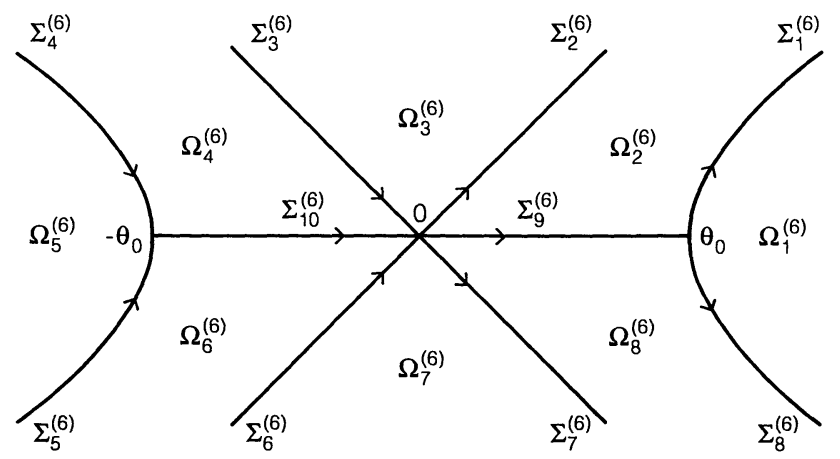

Fig. 6.6. Contour $\Sigma^{(6)}$

Set

$$
\begin{aligned}
& Q^{(6)}(\phi)=Q^{(5)}(\phi), \phi \in \Omega_{1}^{(6)} \cup \Omega_{3}^{(6)} \cup \Omega_{5}^{(6)} \cup \Omega_{7}^{(6)} \\
& Q^{(6)}(\phi)=Q^{(5)}(\phi)\left(\left(b_{+}^{(5)}\right)_{n, t}\right)^{-1}, \phi \in \Omega_{2}^{(6)} \cup \Omega_{4}^{(6)} \\
& Q^{(6)}(\phi)=Q^{(5)}(\phi)\left(\left(b_{-}^{(5)}\right)_{n, t}\right)^{-1}, \phi \in \Omega_{6}^{(6)} \cup \Omega_{8}^{(6)}
\end{aligned}
$$

It now follows immediately, since the jump across parts of $\Sigma^{(6)}$ is $I$, that the problem reduces to one on a simpler contour. In fact, we end up with a Riemann-Hilbert problem on $\Sigma^{(7)}$ (see Fig. 6.7).

Finally, through reorientation and extension (see Fig. 6.8), and with $Q^{(8)}$ defined as follows,

$$
\begin{aligned}
& Q^{(8)}(\phi)=Q^{(7)}(\phi), \phi \in \Omega_{1}^{(8)} \cup \Omega_{2}^{(8)} \cup \Omega_{4}^{(8)} \cup \Omega_{5}^{(8)}, \\
& Q^{(8)}(\phi)=Q^{(7)}(\phi) u_{n, t}^{(7)}(\phi), \phi \in \Omega_{3}^{(8)} \\
& Q^{(8)}(\phi)=Q^{(7)}(\phi)\left(u_{n, t}^{(7)}(\phi)\right)^{-1}, \phi \in \Omega_{6}^{(8)}
\end{aligned}
$$




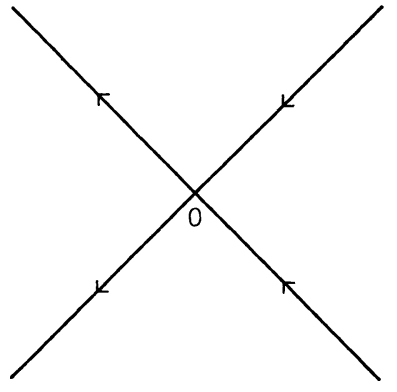

Fig. 6.7

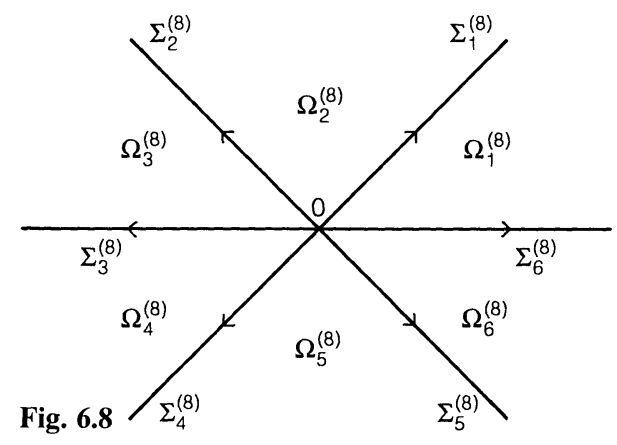

we have the corresponding problem on contour $\Sigma^{(8)}$, with jump matrix

$$
\begin{aligned}
& u_{n, t}^{(8)}(\phi)=e^{-i \Phi a d \sigma_{3}}\left(\begin{array}{cc}
1 & 0 \\
r(0) & 1
\end{array}\right), \phi \in \Sigma_{1}^{(8)}, \\
& u_{n, t}^{(8)}(\phi)=e^{-i \Phi a d \sigma_{3}}\left(\begin{array}{cc}
1 & 0 \\
-r(0) & 1
\end{array}\right), \phi \in \Sigma_{3}^{(8)}, \\
& u_{n, t}^{(8)}(\phi)=e^{-i \Phi a d \sigma_{3}}\left(\begin{array}{cc}
1 & -r(0) \\
0 & 1
\end{array}\right), \phi \in \Sigma_{4}^{(8)} \\
& u_{n, t}^{(8)}(\phi)=e^{-\imath \Phi a d \sigma_{3}}\left(\begin{array}{cc}
1 & r(0) \\
0 & 1
\end{array}\right), \phi \in \Sigma_{6}^{(8)} \\
& u_{n, t}^{(8)}(\phi)=I, \phi \in \Sigma_{2}^{(8)} \cup \Sigma_{5}^{(8)} .
\end{aligned}
$$

The crucial fact (which has motivated the above series of deformations) is that Eqs. (6.19)-(6.20) give the connection with the Painlevé II equation.

Theorem 6.1 (see e.g. [I-N] or [F-N]). Let $u(s)$ solve the Painlevé II equation

$$
u_{s s}-s u-2 u^{3}=0 \text {. }
$$

Consider the associated linear equation

$$
\frac{d \Psi}{d \phi}=\left(\begin{array}{cc}
-4 i \phi^{2}-i s-2 i u^{2} & 4 i u \phi-2 u_{s} \\
-4 i u \phi-2 u_{s} & 4 i \phi^{2}+i s+2 i u^{2}
\end{array}\right) \Psi
$$

and let $\Psi_{1}, \Psi_{2}, \Psi_{3}, \Psi_{4}, \Psi_{5}, \Psi_{6}$ solve (6.22) in the sectors $\Omega_{1}^{(8)}, \Omega_{2}^{(8)}, \Omega_{3}^{(8)}, \Omega_{4}^{(8)}, \Omega_{5}^{(8)}$, $\Omega_{6}^{(8)}$ of Fig. 6.8 with asymptotics given by

$$
\Psi_{\imath} \sim\left(I+\frac{\Psi_{i}^{1}}{\phi}+\frac{\Psi_{\imath}^{2}}{\phi}+\ldots\right) e^{\frac{-4 \imath}{3}\left(\phi^{3}+s \phi\right) \sigma_{3}}, \quad \text { as } \phi \rightarrow \infty
$$

Across each ray $\Sigma_{i}^{(8)}$, one has the following relations (defining the so-called Stokes matrices)

$$
\begin{gathered}
\Psi_{\imath+1}=\Psi_{i} S_{i}, \quad 1 \leq i \leq 5 \\
\Psi_{1}=\Psi_{6} S_{6}
\end{gathered}
$$


where

$$
\begin{aligned}
& S_{1}=\left(\begin{array}{cc}
1 & 0 \\
p & 1
\end{array}\right), \quad S_{2}=\left(\begin{array}{cc}
1 & r \\
0 & 1
\end{array}\right), \quad S_{3}=\left(\begin{array}{cc}
1 & 0 \\
p & 1
\end{array}\right), \\
& S_{4}=\left(\begin{array}{cc}
1 & -p \\
0 & 1
\end{array}\right), \quad S_{5}=\left(\begin{array}{cc}
1 & 0 \\
-r & 1
\end{array}\right), \quad S_{6}=\left(\begin{array}{cc}
1 & -q \\
0 & 1
\end{array}\right) \text {, }
\end{aligned}
$$

$p, q, r$ being complex numbers, independent of $\phi$ and $s$, and satisfying

$$
r=p+q+p q r .
$$

(In fact, $p, q, r$ provide a parametrisation of the 2-dimensional manifold of the solutions of Eq. (6.21).)

Conversely, if relation (6.26) holds, the (unique) solution of (6.23)-(6.25) enables us to recover $u(s)$ as follows:

$$
u=-\lim _{\phi \rightarrow \infty, \phi \in \Omega_{i}^{(8)}}\left(\phi\left[\sigma_{3}, \hat{\Psi}_{i}\right]\right)_{21},
$$

where

$$
\hat{\Psi}_{i} \sim I+\frac{\Psi_{i}^{1}}{\phi}+\frac{\Psi_{i}^{2}}{\phi^{2}}+\ldots
$$

Lemma 6.2. If $\Psi$ is the solution of (6.23)-(6.25) above and

$$
\Psi(0)=\left(\begin{array}{ll}
s_{1} & s_{2} \\
s_{3} & s_{4}
\end{array}\right),
$$

then

$$
\lim _{\operatorname{Im} \phi \rightarrow \infty} Q^{(8)}=\left(\begin{array}{ll}
k_{1}\left(1+s_{2}\right) & k_{1} s_{1} \\
k_{2}\left(1+s_{2}\right) & k_{2} s_{1}
\end{array}\right)
$$

$k_{1}$ and $k_{2}$ being some constants.

Proof. Analogous to the proof of the lemmas in the Appendix, making use of the a priori necessary symmetry of the factorization problem on $\Sigma^{(8)}$.

Applying Theorem 6.1 with

$$
\begin{array}{ll}
p=r(\phi=0), & q=-r(\phi=0), \\
r=0, & s=-2\left(t^{2 / 3}-n t^{-1 / 3}\right),
\end{array}
$$

and the above lemma, we recover $\lim _{\operatorname{Im} \phi \rightarrow \infty} Q^{(8)}(\phi)$ and thus $Q(z=0)$. Keeping in mind formula (A.15) giving $b_{n}$ in terms of $Q(z=0)$ (independently of $k_{1}$ and $k_{2}$ ), we finally obtain formulae $(0.3),(0.5)$.

Remark. The boundedness of the different operators of the form $\left(I-C_{w}\right)^{-1}$ follows in the same way as for the case $n / t<1-C t^{-2+\delta}$. However, one recalls that it depends on the fact that $\sup |r(z)|<1$ (which holds automatically in that region). Near $n / t=1$ this is not necessarily true; in fact $r(1)=-1$ generically. The study of this case requires more work and will be considered in a future paper.

II. We continue with the case $n / t>1$ (near $n / t=1$ ). In this case, the stationary phase points are imaginary (see Fig. 0.2). Our analysis is analogous to that of Sect. I. First, we split $r$ as in (6.2), again through the use of Fourier transforms, where this 
time the Fourier variable is $\Theta=\sin \theta$. We eventually and up with a Riemann-Hilbert problem on the contour shown.

Fig. 6.9

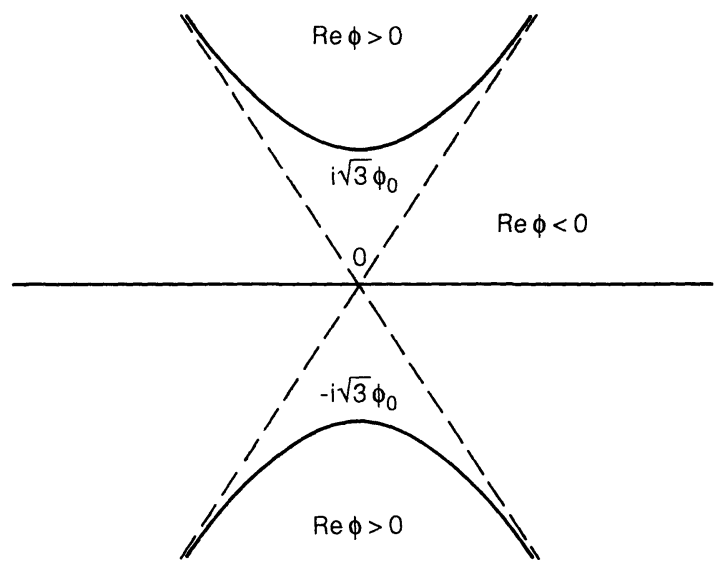

Through an approximation similar to (6.13) we end up once more with problem (6.23)-(6.25). The result follows as in Sect. I.

III. A similar argument shows that in the region $|n / t|>1$, we have $b_{n}-1=0\left(t^{-l}\right)$ for any positive $l$. Indeed, since $s \rightarrow \infty$ as $t \rightarrow \infty$ ( $s$ defined by (6.29)), we see that our final factorization problem on $\Sigma^{(8)}$ has a jump matrix equal to $I$. everywhere. Its solution is thus trivial and the result follows easily.

\section{Appendix. Scattering, Inverse Scattering and a Riemann-Hilbert Matrix Factorization Problem}

As is well known, the Toda lattice is a (formally) completely integrable Hamiltonian system which can be solved by the use of the inverse scattering transform (see [G, O].) In this appendix, we present some of the facts about this method of solution that are needed in the main part of this paper.

We begin with the analysis of the scattering and inverse scattering for the discrete Lax operator $L$ (the analogue of the Schroedinger operator in the KdV case). It is defined on the Hilbert space $l^{2}$ as follows:

$$
(L u)_{n}=b_{n-1} u_{n-1}+a_{n} u_{n}+b_{n} u_{n+1},
$$

where $a_{n}$ and $b_{n}-1 / 2$ are decaying faster than any polynomial as $n \rightarrow \infty$.

It is easy to see that the continuous spectrum of $L$ consists of the band $[-1,1]$ with multiplicity 2 . In this paper, we will assume that the discrete spectrum is empty. (This means that no solitons are allowed; they will be added in a later study.)

Define the Jost functions as follows (see [F]): let $\phi(n, z)$ and $\psi(n, z)$ be such that

$$
\begin{array}{lll}
L \phi(n, z)=\lambda \phi(n, z) ; & \phi(n, z) \sim z^{n} & \text { as } n \rightarrow \infty, \\
L \psi(n, z)=\lambda \psi(n, z) ; & \psi(n, z) \sim z^{-n} & \text { as } n \rightarrow-\infty .
\end{array}
$$

Here the spectral variable $z$ is defined by

$$
2 \lambda=z+1 / z \text {. }
$$


The band $[-1,1]$ in the $\lambda$-plane with multiplicity 2 is thus mapped to the unit circle in the $z$-plane with multiplicity 1 .

Next, let $B_{n}^{+}=\prod_{k=n}^{\infty}\left(2 b_{k}\right)^{-1}, B_{n}^{-}=\prod_{k=-\infty}^{n-1}\left(2 b_{k}\right)^{-1}$ and $B=B_{n}^{+} B_{n}^{-}$. The following facts are proved in $[\mathrm{O}]$.

We can write

$$
\phi(n, z)=B_{n}^{+} z^{n} v_{n}(z), \quad \psi(n, z)=B_{n}^{+} z^{-n} u_{n}(z),
$$

where $v_{n}(z)=1+\sum_{n=1}^{\infty} v_{n, k} z^{k}$ and $u_{n}(z)=1+\sum_{n=1}^{\infty} u_{n, k} z^{k}$, both series converging uniformly in $|z| \leq 1$.

From (A.2) we can derive the following recursive relation:

$$
\begin{aligned}
v_{n}(z)= & 1-\sum_{j=n+1}^{\infty} \frac{1}{1-z^{2}}\left(2 a_{j} z v_{j}(z)+\left(4 b_{n}^{2}-1\right) z^{2} v_{j+1}(z)\right) \\
& -\sum_{j=n+1}^{\infty} \frac{z^{2}(j-n)}{1-z^{2}}\left(2 a_{j} z v_{j}(z)+\left(4 b_{n}^{2}-1\right) z^{2} v_{j+1}(z)\right) .
\end{aligned}
$$

As $n \rightarrow-\infty$, we get

$$
\begin{aligned}
v_{n}(z) & 1-\sum_{j=-\infty}^{\infty} \frac{1}{1-z^{2}}\left(2 a_{j} z v_{\jmath}(z)+\left(4 b_{n}^{2}-1\right) z^{2} v_{j+1}(z)\right) \\
& -z^{-2 n} \sum_{j=-\infty}^{\infty} \frac{z^{2 j}}{1-z^{2}}\left(2 a_{j} z v_{j}(z)+\left(4 b_{n}^{2}-1\right) z^{2} v_{j+1}(z)\right) .
\end{aligned}
$$

On the other hand, when $z \neq \pm 1, \phi(n, z), \psi(n, z)$ and $\phi\left(n, z^{-1}\right), \psi\left(n, z^{-1}\right)$ are two sets of independent solutions of the second order difference equation $L \chi=\lambda \chi$. Hence there exist $A(z), B(z), \alpha(z), \beta(z)$ such that

$$
\begin{aligned}
& \phi(n, z)=B(z) \psi(n, z)+A(z) \psi\left(n, z^{-1}\right), \quad z \in \mathbb{C}-\{-1,1\} . \\
& \psi(n, z)=\beta(z) \phi(n, z)+\alpha(z) \phi\left(n, z^{-1}\right),
\end{aligned}
$$

Making use of (A.3) and (A.5) we get

$$
v_{n}(z)=\frac{B_{n}^{-}}{B_{n}^{+}} B(z) z^{-2 n} u_{n}(z)+\frac{B_{n}^{-}}{B_{n}^{+}} A(z) u_{n}\left(z^{-1}\right) .
$$

As $n \rightarrow-\infty, B_{n}^{-} \rightarrow 1, B_{n}^{+} \rightarrow B, u_{n}(z) \rightarrow 1, u_{n}\left(z^{-1}\right) \rightarrow 1$. Hence

$$
B v_{n}(z) \sim B(z) z^{-2 n}+A(z) .
$$

Using the above recursion relation (A.4) for $v_{n}(z)$ we get

$$
\begin{aligned}
& \frac{B(z)}{B}=-\sum_{j=-\infty}^{\infty} \frac{z^{2 j}}{1-z^{2}}\left(2 a_{j} z v_{j}(z)+\left(4 b_{n}^{2}-1\right) z^{2} v_{j+1}(z)\right), \\
& \frac{A(z)}{B}=-\sum_{j=-\infty}^{\infty} \frac{1}{1-z^{2}}\left(2 a_{j}(z)+\left(4 b_{n}^{2}-1\right) z^{2} v_{j+1}(z)\right) .
\end{aligned}
$$


Define $T(z)=\frac{1}{A(z)}$ and $R(z)=\frac{\beta(z)}{\alpha(z)}$. Then

$$
\frac{1}{B T(z)}=1-\sum_{\jmath=-\infty}^{\infty} \frac{1}{1-z^{2}}\left(2 a_{j}(z)+\left(4 b_{n}^{2}-1\right) z^{2} v_{\jmath+1}(z)\right)
$$

for $|z| \leq 1$. Thus, $T(z)$ is defined not only on $|z|=1$, but is also extended meromorphically to $|z|<1$, and in fact $T(0)=1 / B$. Our assumption that no bound states exist is in fact equivalent to the fact that $T(z)$ in indeed holomorphic in $|z|<1$.

We note here that in general, the reflection coefficient $R(z)$ cannot be extended meromorphically unless the decay of $a_{n}$ and $b_{n}-1 / 2$ is exponential.

Next define

$$
U_{1}(n, z)=\psi(n, z), \quad U_{2}(n, z)=\frac{\phi(n, z)}{A(z)}=T(z) \phi(n, z) .
$$

Since $\phi(n, z) \sim B_{n}^{+} z^{n}$ and $\psi(n, z) \sim B_{n}^{-} z^{-n}$ near $z=0$, we have

$$
\begin{aligned}
& U_{1}(n, z) \sim B_{n}^{-} z^{-n}, \quad \text { near } z=0 . \\
& U_{2}(n, z) \sim B_{n}^{+} z^{n},
\end{aligned}
$$

The following relations are easy to prove:

$$
B(z)=\bar{B}(z), \quad A\left(z^{-1}\right)=\bar{A}(z), \quad|R(z)|^{2}+|T(z)|^{2}=1 .
$$

Hence $\alpha(z) A\left(z^{-1}\right)=|A(z)|^{2}=\frac{1}{|T(z)|^{2}}=\frac{1}{1-|R(z)|^{2}}$.

We can now rewrite (A.5) as

$$
\begin{aligned}
& U_{1}(n, z)=-B(z) A(z) U_{2}(n, z)+|A(z)|^{2} U_{2}\left(n, z^{-1}\right), \\
& U_{2}(n, z)=\frac{B(z)}{A(z)} U_{1}(n, z)+U_{1}\left(n, z^{-1}\right) .
\end{aligned}
$$

By their definitions, and by the properties of the functions $\phi$ and $\psi$ (see discussion after A.3) we can extend $U_{1}$ and $U_{2}$ inside, but also outside the unit circle (extending $U_{1}\left(n, z^{-1}\right)$ and $\left.U_{2}\left(n, z^{-1}\right)\right)$. On the unit circle we denote the inner normal limit of $U_{1}$ by $U_{1}^{+}$and the normal limit from outside by $U_{1}^{-}$(and similarly with $U_{2}$ ). Defining $r(z)=\frac{B(z)}{A(z)}$, we finally get

$$
\left(U_{2}^{+}, U_{1}^{+}\right)=\left(U_{2}^{-}, U_{1}^{-}\right)\left(\begin{array}{cc}
1-|r(z)|^{2} & -\bar{r}(z) \\
r(z) & 1
\end{array}\right)
$$

with asymptotics

$$
\left(U_{1}, U_{2}\right)=\left(B_{n}^{-} z^{-n},\left(B_{n}^{-}\right)^{-1} z^{n}\right)
$$

at $z=0$ and

$$
\left(U_{1}, U_{2}\right) \sim\left(B_{n}^{-} z^{n},\left(B_{n}^{-}\right)^{-1} z^{n}\right)
$$

at $z=\infty$. 


\section{Defining}

$$
\begin{aligned}
& y_{1}(n, z)=\frac{U_{2}(n, z)}{z^{n}} \quad \text { when }|z|<1, \\
& y_{1}(n, z)=\frac{U_{1}(n, z)}{z^{n}} \quad \text { when }|z|>1, \\
& y_{2}(n, z)=\frac{U_{2}(n, z)}{z^{-n}} \quad \text { when }|z|<1, \\
& y_{2}(n, z)=\frac{U_{2}(n, z)}{z^{-n}} \quad \text { when }|z|>1,
\end{aligned}
$$

and letting $Y=\left(y_{1}, y_{2}\right)$, we end up with the Riemann-Hilbert matrix factorization problem:

$$
Y_{+}=Y_{-}\left(\begin{array}{cc}
1-|r(z)|^{2} & -r(z) z^{2 n} \\
r(z) z^{-2 n} & 1
\end{array}\right)
$$

with asymptotics at $\infty$ :

$$
Y(\infty)=\left(\left(B_{n}^{-}\right)^{-1}, B_{n}^{-}\right)
$$

Furthermore,

$$
Y(0)=\left(B_{n}^{-},\left(B_{n}^{-}\right)^{-1}\right) .
$$

Note that the solution of (A.8)-(A.9) is unique as follows easily by Liouville's theorem (see Theorem 1.2).

As is easy to see (see [O]), during the Toda flow the evolution of the reflection coefficient with time is given by

$$
r(z)=r(z, t)=r(z, 0) \exp \left(t\left(z-z^{-1}\right)\right) .
$$

So, at time $t$, relation (A.8) becomes

$$
Y_{+}(z, t)=Y_{-}(z, t)\left(\begin{array}{cc}
1-|r(z, 0)|^{2} & -\bar{r}(z, 0) z^{2 n} e^{-t\left(z-z^{-1}\right)} \\
r(z, t) z^{-2 n} e^{t\left(z-z^{-1}\right)} & 1
\end{array}\right) .
$$

We next proceed to the inverse scattering problem: given the solution of (A.8)-(A.9), what is the solution of the Cauchy problem (0.1)-(0.2)?

As the Riemann-Hilbert problem for $Y$ has an inconvenient condition at infinity, we will reduce it to the one as follows: let $Q(z)$ be analytic in $\mathbb{C}-C$, with normal limits $Q_{+}$and $Q_{-}$on $C$, satisfying

$$
Q_{+}=Q_{-} u_{n, t}, \quad Q(\infty)=I
$$

where $u_{n, t}$ is the $2 \times 2$ matrix appearing in (A.8) [recall Definition (0.8)].

Theorem. Let

$$
Q(0)=\left(\begin{array}{ll}
\alpha & \beta \\
\gamma & \delta
\end{array}\right)
$$

say, and

$$
Y=\left(\left(\frac{1+\beta}{\alpha}\right)^{1 / 2}\left(\frac{\alpha}{1+\beta}\right)^{1 / 2}\right) Q .
$$

Then $Y$ solves (A.8)-(A.9), up to a constant scalar multiple. 
Proof. Clearly (A.8) holds. On the other hand we have the following lemmas.

Lemma 1. $Q(z)=\left(\begin{array}{ll}0 & 1 \\ 1 & 0\end{array}\right)(Q(0))^{-1} Q\left(z^{-1}\right)\left(\begin{array}{ll}0 & 1 \\ 1 & 0\end{array}\right)$.

Proof. We observe that

$$
v(z)=\left(\begin{array}{ll}
0 & 1 \\
1 & 0
\end{array}\right)\left(v\left(z^{-1}\right)\right)^{-1}\left(\begin{array}{ll}
0 & 1 \\
1 & 0
\end{array}\right)
$$

Thus,

$$
Q_{+}(z)=Q_{-}(z)\left(\begin{array}{ll}
0 & 1 \\
1 & 0
\end{array}\right)\left(v\left(z^{-1}\right)\right)^{-1}\left(\begin{array}{ll}
0 & 1 \\
1 & 0
\end{array}\right)
$$

hence

$$
\left(\begin{array}{ll}
0 & 1 \\
1 & 0
\end{array}\right) Q_{+}(z)\left(\begin{array}{ll}
0 & 1 \\
1 & 0
\end{array}\right)=\left(\begin{array}{ll}
0 & 1 \\
1 & 0
\end{array}\right) Q_{-}(z)\left(\begin{array}{ll}
0 & 1 \\
1 & 0
\end{array}\right)\left(v\left(z^{-1}\right)\right)^{-1}
$$

Applying this to $z^{-1}$,

$$
\left(\begin{array}{ll}
0 & 1 \\
1 & 0
\end{array}\right) Q_{+}\left(z^{-1}\right)\left(\begin{array}{ll}
0 & 1 \\
1 & 0
\end{array}\right) v(z)=\left(\begin{array}{ll}
0 & 1 \\
1 & 0
\end{array}\right) Q_{-}\left(z^{-1}\right)\left(\begin{array}{ll}
0 & 1 \\
1 & 0
\end{array}\right)
$$

Defining

$$
H(z)=\left(\begin{array}{ll}
0 & 1 \\
1 & 0
\end{array}\right) Q\left(z^{-1}\right)\left(\begin{array}{ll}
0 & 1 \\
1 & 0
\end{array}\right)
$$

we have

$$
H_{+}(z)=H_{-}(z) v(z)
$$

and thus (by Liouville's theorem)

$$
H(z)=A^{-1} Q(z)
$$

for some constant invertible matrix $A$. Thus,

$$
Q(z)=A\left(\begin{array}{ll}
0 & 1 \\
1 & 0
\end{array}\right) Q\left(z^{-1}\right)\left(\begin{array}{ll}
0 & 1 \\
1 & 0
\end{array}\right) .
$$

Letting $z \rightarrow \infty$, we get

$$
I=A\left(\begin{array}{ll}
0 & 1 \\
1 & 0
\end{array}\right) Q(0)\left(\begin{array}{ll}
0 & 1 \\
1 & 0
\end{array}\right),
$$

which gives $A$. The lemma now follows from (A.13).

It follows from Lemma 1 that for $Y$ defined by (A.12) we have

$$
Y(z)=Y\left(z^{-1}\right)\left(\begin{array}{ll}
0 & 1 \\
1 & 0
\end{array}\right) .
$$

Lemma 2. If $Y$ satisfies (A.8) and (A.14) then $Y$ also satisfies (A.9), up to a scalar multiple. 
Proof. We show that there is only one solution of (A.8) satisfying the symmetry condition (A.14). In fact, suppose there is a second one, say $X$. Consider the matrix

$$
Z=\left(\begin{array}{l}
Y \\
X
\end{array}\right)
$$

Then, $Z_{+}=Z_{-} v$, hence $\operatorname{det} Z_{+}=\operatorname{det} Z_{-} \operatorname{det} v=\operatorname{det} Z_{-}$, and by Liouville's theorem, $\operatorname{det} Z$ is constant, say $c$. On the other hand, by assumption,

$$
M(z)=M\left(z^{-1}\right)\left(\begin{array}{ll}
0 & 1 \\
1 & 0
\end{array}\right),
$$

and, taking determinants,

$$
c=c(-1)
$$

hence $c=0$, thus $X$ and $Y$ are dependent. This proves Lemma 2, and the theorem also follows.

Lemma 3. If

$$
Q(0)=\left(\begin{array}{ll}
\alpha & \beta \\
\gamma & \delta
\end{array}\right)
$$

then $\beta=-\gamma$, and $\alpha \delta-\beta \gamma=1$.

Proof. By a Liouville's-theorem-argument as in Lemma 2, it follows that $\operatorname{det} Q$ is constant, and in fact 1, from which the second assertion of Lemma 3 follows. The first assertion follows immediately, by setting $z=0$ in Lemma 1 .

A direct consequence of the theorem above together with Lemma 3 is that the solution of (A.8)-(A.9) satisfies

$$
Y(0)=k\left(\left(\frac{\alpha}{1+\beta}\right)^{1 / 2}\left(\frac{1+\beta}{\alpha}\right)^{1 / 2}\right),
$$

for some constant $k$. Comparing with (A.9) we get $k=1$, and

$$
B_{n}^{-}=\left(\frac{\alpha}{1+\beta}\right)^{1 / 2}
$$

from which $b_{n}$ can be recovered by dividing.

Thus we have reduced our Cauchy problem (0.1)-(0.2) to the Riemann-Hilbert problem (A.11) and in particular to finding the first row of $Q(0)$.

Acknowledgements. I wish to thank Percy Deift for suggesting the problem, for helpful discussions and suggestions, and more specifically for the proofs of the lemmas of the appendix.

This research was supported by a Scholarship from the Onassis Foundation of Athens, Greece, a Postdoctoral Fellowship of the French Government and a Department of Energy grant, no. DE-FG02-88ER25053. 


\section{References}

[B-C] Beals, R., Coifman, R.: Scattering and inverse scattering for first order systems. Commun. Pure App. Math. 37, 39-90 (1984)

[D-Z] Deift, P.A., Zhou, A.: A Steepest descent method for oscillatory Riemann-Hilbert problems. To appear

[F] Flaschka, H.: On the Toda lattice. II. Progr. Theor. Phys. 51/3, 703-716 (1974)

[F-N] Flaschka, H., Newell, A.C.: Monodromy- and spectrum-preserving transformations. I. Commun. Math. Phys. 76, 67-116 (1980)

[G] Guseinov, G.S.: The inverse problem of scattering theory for a second-order difference equation on the whole axis. Sov. Math. Doklady 17/4, 1684-1688 (1976)

[I] Its, A.R.: Asymptotics of solutions of the nonlinear Schrödinger equation and isomonodromic deformations of systems of linear differential equations. Sov. Math. Doklady 24/3, 452-456 (1982)

[I-N] Its, A.R., Novokshenov, V.Yu.: The isomonodromic deformation method in the theory of Painlevé equations. Lect. Notes in Math. 1191. Berlin, Heidelberg, New York: Springer 1986

$[\mathrm{N}-\mathrm{H}]$ Novokshenov, V.Yu., Habibullin, I.T.: Nonlinear differential-difference schemes integrable by the method of the inverse scattering problem. Asymptotics of the solution for $t \rightarrow \infty$. Sov. Math. Doklady 23/2, 304-307 (1981)

[O] Oba, R.: Dissertation, Courant Institute, 1988

Communicated by A. Jaffe 
